\title{
Interpretation of dynamic scattering experiments on ternary polymer mixtures
}

\author{
M. Tombakoglu and A. Z. Akcasu* \\ Department of Nuclear Engineering, The University of Michigan, \\ Ann Arbor, MI 48109, USA \\ (Received 11 February 1991; revised 25 April 1991; accepted 28 April 1991)
}

\begin{abstract}
The dynamics of a ternary polymer mixture consisting of either homopolymers A and $\mathrm{B}$, or diblock copolymers $\mathrm{A}-\mathrm{B}$, in a matrix of homopolymers $\mathrm{C}$, is studied using the random phase approximation and assuming incompressibility. The relaxation frequencies and the amplitudes of the modes in dynamic scattering on such a mixture have been calculated as a function of the wave number $q$. In addition, the first cumulant of the dynamic scattering function of a labelled component is obtained in all $q$ regions including the high $q$ region where the segmental diffusion dominates the relaxation of the scattering function. The particular mixture consisting of A-B diblock copolymers in a matrix of homopolymers A, studied recently by Stühn and Rennie, is analysed in detail, by calculating the first cumulant in the entire $q$ region.
\end{abstract}

(Keywords : dynamic scattering ; random phase approximation; ternary polymer mixtures; Rouse dynamics; first cumulant)

\section{INTRODUCTION}

The dynamics of ternary polymer mixtures has been the subject of several experimental and theoretical studies in recent years. On the experimental side, Nemota et al. ${ }^{1}$ studied a binary mixture of polystyrene fractions in benzene in the semidilute regime with dynamic light scattering. Borsali et al. ${ }^{2}$ investigated the dynamics of a mixture consisting of polystyrene/poly (methyl methacrylate)/toluene also by quasielastic light scattering in the semidilute regime. More recently Brown et $\mathrm{al}^{3}$ reported the results of dynamic light scattering from various homopolymer species in solutions. On the other hand Stühn and Rennie ${ }^{4}$ studied the segmental dynamics of styrene-isoprene diblock copolymer in deuterated polyisoprene matrix with spin-echo neutron scattering.

On the theoretical side, the general theory of multicomponent systems was formulated by Akcasu et al. ${ }^{5}$ in 1984 using linear response theory. They showed that in two-component polymer solutions, the dynamic scattering function is expressed as a superposition of two exponential modes (bimodal relaxation), and obtained the exponents and amplitudes of modes explicitly, from which they calculated the apparent diffusion coefficient for the mixture as a function of concentration in the dilute regime. They compared their theoretical predictions to the experimental results on a solution consisting of polystyrene molecules with different molecular weights in cyclohexane at the $\theta$ point. The same formalism was used by Akcasu et al. ${ }^{6}$ to study the dynamics of polyelectrolyte solutions. In $1986 \mathrm{Akcasu}$ et al. ${ }^{7}$ investigated the dynamics of binary homopolymer blends and copolymer melts using Random Phase Approximation (RPA), and calculated the first cumulant of the dynamic

* To whom correspondence should be addressed at: Facultat für Physik, Universität Konstanz, Postfach 5560, D-7750 Konstanz, Germany scattering function as a function of wavelength. In 1987 Benmouna et $a l .{ }^{8}$ rederived the earlier results in reference 5 , using the linear response theory to calculate the mobilities without including hydrodynamic interactions, and the RPA to calculate the static structure factors, and then analysed the experiments by Nemota et al. ${ }^{1}$. Also, in 1987 Benmouna et al. ${ }^{9}$ studied the dynamics of copolymers in solvent. Foley and Cohen ${ }^{10}$, used the Flory-Huggins model to describe the dynamics of concentration fluctuations and spinodal decomposition for the ternary system of two polymers and solvent. Stühn and Rennie ${ }^{4}$ extended the results of Akcasu et al. ${ }^{7}$ for diblock copolymer melts, by including the effect of a matrix consisting of one of the species in the copolymers, in order to interpret their spin-echo experiments. More recently Akcasu and Tombakoglu ${ }^{11}$ have developed a general theory of multicomponent incompressible polymer mixtures within the framework of RPA, and obtained general expressions for the dynamic scattering function and its first cumulant in the interacting system in terms of the bare system parameters.

The purpose of this paper is to present a theoretical interpretation of dynamic scattering experiments on ternary systems consisting of three polymer species A, B and $C$ by explicitly calculating the decay constants and amplitudes of the exponential modes, as a special case of the general results we obtained earlier ${ }^{11}$. Since in some experiments, such as the one by Stühn and Rennie ${ }^{4}$, the segmental diffusion is investigated through the first cumulant, we also present the calculation of the first cumulant for all values of wave number $q$ as a function of the interaction parameters in the ternary system. The species A and B are allowed to be the two parts of diblock copolymers or just two distinct homopolymers. When the $\mathrm{C}$ component is chemically identical to the component $\mathrm{A}$ in the case of diblock copolymers, the ternary system reduces to the one studied by Stühn and Rennie ${ }^{4}$. 
However, our expression for the first cumulant of the scattering function, when the A component in the diblock copolymers is assumed to be labelled, is somewhat different from the one used by Stühn and Rennie ${ }^{4}$. We therefore reanalyse their experiment with the theoretical results obtained in this paper in detail, and discuss the information that can be extracted from spin-echo neutron scattering experiments by analysing the first cumulant in the high $q$ region. When the matrix $\mathrm{C}$ is treated as a solvent, we obtain the ternary system studied by Benmouna et al..$^{9}$ although the RPA may be questionable in this limit.

We present the theoretical results for ternary systems and point out the differences between our results and those reported earlier. Then we calculate the first cumulant explicitly as a function of $q$ in various $q$ regions and for different Flory interaction parameters $\kappa$. We also compare the first cumulant in the high $q$ region to the experimental results obtained by Stühn and Rennie ${ }^{4}$, and investigate the variation of the decay rates and the amplitudes of the modes as a function of $q$ under conditions of their experiment. We also present the phase diagram for this particular mixture and discuss variation of the critical wave number $q_{\mathrm{c}}$, associated with microphase separation as a function of the volume fraction of the copolymers.

\section{THEORETICAL FOUNDATIONS}

In incompressible ternary systems the short time behaviour of the scattering intensity from a labelled component, which we denote by A, can be expressed as ${ }^{5}$ :

$$
S_{\mathrm{aa}}(q, t)=a_{1}(q) \mathrm{e}^{-\lambda_{1}(q) t}+a_{2}(q) \mathrm{e}^{-\lambda_{2}(q) t}
$$

where $\lambda_{1}(q)$ and $\lambda_{2}(q)$ are obtained by solving the quadratic equation

$$
\left(\Omega_{11}-\lambda\right)\left(\Omega_{22}-\lambda\right)-\Omega_{12} \Omega_{21}=0
$$

and the amplitudes of each mode are given by ${ }^{5}$ :

$$
\begin{aligned}
& a_{1}(q)=\frac{S_{\mathrm{aa}}(q) \lambda_{2}(q)-q^{2} k_{\mathrm{B}} T m_{\mathrm{aa}}}{\lambda_{2}(q)-\lambda_{1}(q)} \\
& a_{2}(q)=\frac{S_{\mathrm{aa}}(q) \lambda_{1}(q)-q^{2} k_{\mathrm{B}} T m_{\mathrm{aa}}}{\lambda_{1}(q)-\lambda_{2}(q)}
\end{aligned}
$$

In equation (2), $\Omega_{i j}(q)$ denote the elements of the first cumulant matrix $\mathbf{\Omega}(q)$, which must be specified to determine the decay constants. In equation (3), $m_{\mathrm{aa}}$ is a component of the mobility matrix $\mathbf{m}(q)=\left[m_{i j}(q)\right]$. The latter is related to the static structure matrix $\mathbf{S}(q)=$ $\left[S_{i j}(q)\right]$ and $\mathbf{\Omega}(q)$ by

$$
\boldsymbol{\Omega}(q)=q^{2} k_{\mathbf{B}} T \mathbf{m}(q) \mathbf{S}^{-1}(q)
$$

Hence, the problem of calculating $S_{\mathrm{aa}}(q, t)$ is reduced to the calculation of the mobility matrix $m(q)$ and static structure matrix $\mathbf{S}(q)$. The RPA enables one to express these quantities in terms of the bare system mobility matrix $\mathbf{m}^{\circ}(q)$ and bare system static structure matrix $\mathbf{S}^{\circ}(q)$, which are approximated by single chain mobilities and static structure factors. In the case of a ternary mixture of $\mathrm{A}, \mathrm{B}$ and $\mathrm{C}$, in which component $\mathrm{C}$ is assumed to be a homopolymer, treated as the matrix and eliminated using incompressibility, we obtained ${ }^{11}$ the matrix elements $m_{i j}(q)$ :

$$
\frac{1}{m_{\mathrm{aa}}}=\frac{1}{m_{\mathrm{aa}}^{\mathrm{o}}}+\frac{1}{m_{\mathrm{bb}}^{\mathrm{o}}+m_{\mathrm{cc}}^{\mathrm{o}}}
$$

$$
\begin{aligned}
\frac{1}{m_{\mathrm{bb}}} & =\frac{1}{m_{\mathrm{bb}}^{\mathrm{o}}}+\frac{1}{m_{\mathrm{aa}}^{\mathrm{o}}+m_{\mathrm{cc}}^{\mathrm{o}}} \\
\frac{1}{m_{\mathrm{ab}}} & =-\left(\frac{1}{m_{\mathrm{aa}}^{\mathrm{o}}}+\frac{1}{m_{\mathrm{bb}}^{\mathrm{o}}}+\frac{m_{\mathrm{cc}}^{\mathrm{o}}}{m_{\mathrm{aa}}^{\mathrm{o}} m_{\mathrm{bb}}^{\mathrm{o}}}\right)
\end{aligned}
$$

The mobilities $m_{i j}^{o}$ in the bare system are customarily calculated by using Rouse dynamics, as

$$
m_{\mathrm{aa}}^{\mathrm{o}}=\frac{\phi_{\mathrm{a}}}{\zeta_{\mathrm{a}}}, \quad m_{\mathrm{bb}}^{\mathrm{o}}=\frac{\phi_{\mathrm{b}}}{\zeta_{\mathrm{b}}}, \quad m_{\mathrm{cc}}^{\mathrm{o}}=\frac{\phi_{\mathrm{c}}}{\zeta_{\mathrm{c}}}
$$

where $\phi_{i}$ is the volume fraction of each component, and defined as the ratio of the total number of segments of species to the total number of segments in the entire system, assuming that the segmental volumes are the same. We note that the bare mobilities can be expressed in terms of the friction coefficients $\zeta_{i}$ with different proportionality constants from the volume fractions used in equation (6), such as the total number of monomers in the components. The choice depends on the normalization used in the definition of the static structure factors. Since the first cumulant is expressed as the ratio of the mobilities and static structure factors as can be seen in equation (4), it is independent of the choice of their normalization. In the case of a ternary system consisting of copolymers $\mathbf{A}-\mathbf{B}$ in the matrix of homopolymers $\mathbf{C}$, these volume fractions are expressed as $\phi_{\mathrm{a}}=f_{\mathrm{a}} \phi, \phi_{\mathrm{b}}=$ $\left(1-f_{\mathrm{a}}\right) \phi$ and $\phi_{\mathrm{c}}=(1-\phi)$, where $\phi$ is the volume fraction of all the segments belonging to the copolymers, and $f_{\mathrm{a}}$ is the fraction of $\mathrm{A}$ segments in copolymer $\mathrm{A}-\mathrm{B}$. The description of single chain dynamics in the polymer mixture by the Rouse model is a simplification suggested and employed by de Gennes ${ }^{12}$, Pincus ${ }^{13}$ and Binder ${ }^{14}$, which we also adopted in this and our earlier papers ${ }^{7}$ on the dynamics of polymer mixtures. It may be justifiable in a mixture of polymers, in which the hydrodynamic interactions are fully screened. It is however possible to accommodate reptation, by choosing the bare mobilities as $m_{\alpha \alpha}^{\circ}=\phi_{\alpha} / \zeta_{\alpha} N_{\alpha}$, where $N_{\alpha}$ is the number of monomers in $\alpha$ chain.

The matrix elements of the structure matrix $\mathbf{S}(q)$, needed in equation ( 3 ), are calculated using the inverse Zimm formula in matrix form ${ }^{15}$ :

$$
\mathbf{S}^{-1}(q)=\mathbf{S}^{\mathbf{o}^{-1}}(q)+\mathbf{v}(q)
$$

where the third component $\mathrm{C}$ has already been eliminated by using incompressibility constraint so that $\mathbf{S}(q), \mathbf{S}^{\circ}(q)$ and $\mathbf{v}(q)$ in equation $(7)$ are $2 \times 2$ square matrices. The elements of excluded volume matrix $v(q)$ are given ${ }^{11,16}$ by:

$$
\begin{aligned}
v_{\mathrm{aa}}(q) & =\frac{1}{S_{\mathrm{cc}}^{\mathrm{o}}(q)}-2 \kappa_{\mathrm{ac}} \\
v_{\mathrm{ab}}(q) & =\frac{1}{S_{\mathrm{cc}}^{\mathrm{o}}(q)}+\kappa_{\mathrm{ab}}-\kappa_{\mathrm{ac}}-\kappa_{\mathrm{bc}} \\
\kappa_{\mathrm{ab}} & =\frac{1}{k_{\mathrm{b}} T v_{\mathrm{s}}}\left[W_{\mathrm{ab}}-\frac{1}{2}\left(W_{\mathrm{aa}}+W_{\mathrm{bb}}\right)\right]
\end{aligned}
$$

and $v_{\mathrm{bb}}(q)$ is obtained replacing $\kappa_{\mathrm{ac}}$ by $\kappa_{\mathrm{bc}}$ in equation (8a). In equation $(8 \mathrm{c}), v_{\mathrm{s}}$ is the segmental volume, which is assumed to be the same for all species, and $W_{\mathrm{ab}}(q)$ denotes the Fourier transform of the interaction potential per particle of a pair of A and B monomers, and has a unit of volume-energy. Hence, $\kappa_{i j}$ are dimensionless, and the excluded volume parameters are expressed per unit 
segmental volume so that they are also dimensionless. The normalization of $S_{\mathrm{cc}}^{\mathrm{o}}(q)$ in equation (8) is such that $S_{\mathrm{cc}}^{\mathrm{o}}(0)=\phi_{\mathrm{c}} N_{\mathrm{c}}$, where $N_{\mathrm{c}}$ denotes the number of monomers in a matrix chain. These normalizations are consistent with the normalization of mobilities introduced in equation (6). The elements of the static structure matrix can be obtained ${ }^{11,16}$ explicitly from equation (7) as

\section{$\mathbf{S}(q)=$}

$\frac{1}{\Delta}\left[\begin{array}{c}\left(S_{\mathrm{aa}}^{\mathrm{o}} S_{\mathrm{cc}}^{o}+\Delta^{\mathrm{o}}-2 \kappa_{\mathrm{bc}} S_{\mathrm{cc}}^{\mathrm{o}} \Delta^{\circ}\right)\left(S_{\mathrm{ab}}^{\mathrm{o}} S_{\mathrm{cc}}^{\mathrm{o}}-\Delta^{\mathrm{o}}-\kappa_{\mathrm{abc}} S_{\mathrm{cc}}^{\mathrm{o}} \Delta^{\mathrm{o}}\right) \\ \left(S_{\mathrm{ab}}^{\mathrm{o}} S_{\mathrm{cc}}^{\mathrm{o}}-\Delta^{\mathrm{o}}-\kappa_{\mathrm{abc}} S_{\mathrm{cc}}^{\mathrm{o}} \Delta^{\mathrm{o}}\right)\left(S_{\mathrm{bb}}^{\mathrm{o}} S_{\mathrm{cc}}^{\mathrm{o}}+\Delta^{\mathrm{o}}-2 \kappa_{\mathrm{ac}} S_{\mathrm{cc}}^{\mathrm{o}} \Delta^{\circ}\right)\end{array}\right]$

where

$$
\begin{gathered}
\Delta=S_{\mathrm{aa}}^{\mathrm{o}}+S_{\mathrm{bb}}^{\mathrm{o}}+2 S_{\mathrm{ab}}^{\mathrm{o}}+S_{\mathrm{cc}}^{\mathrm{o}}-2 \kappa_{\mathrm{ac}}\left(S_{\mathrm{aa}}^{\mathrm{o}} S_{\mathrm{cc}}^{\mathrm{o}}+\Delta^{\circ}\right) \\
-2 \kappa_{\mathrm{bc}}\left(S_{\mathrm{bb}}^{\mathrm{o}} S_{\mathrm{cc}}^{\mathrm{o}}+\Delta^{\mathrm{o}}\right)-2 \kappa_{\mathrm{abc}}\left(\Delta^{\mathrm{o}}-S_{\mathrm{ab}}^{\mathrm{o}} S_{\mathrm{cc}}^{\mathrm{o}}\right) \\
+\left(4 \kappa_{\mathrm{ac}} \kappa_{\mathrm{bc}}-\kappa_{\mathrm{abc}}^{2}\right) S_{\mathrm{cc}}^{\mathrm{o}} \Delta^{\mathrm{o}} \\
\kappa_{\mathrm{abc}}=\kappa_{\mathrm{ab}}-\kappa_{\mathrm{ac}}-\kappa_{\mathrm{bc}} \\
\Delta^{\circ}=S_{\mathrm{aa}}^{\mathrm{o}} S_{\mathrm{bb}}^{\mathrm{o}}-S_{\mathrm{ab}}^{\mathrm{o} 2}
\end{gathered}
$$

In these equations $S_{\mathrm{aa}}^{\mathrm{o}}(q)$ and $S_{\mathrm{bb}}^{\mathrm{o}}(q)$ are the single chain static structure factors of $A$ and $B$ components in the mixture. In the bulk state they obey Gaussian statistics, and are usually represented by the Debye function, in which $q a \ll 1$ is assumed. Here ' $a$ ' denotes the statistical segment length of an A chain. Since Stühn and Rennie's experiment is performed in the high $q$ region, where $q a \sim 1$, we need the full expression of $S_{\mathrm{aa}}^{\mathrm{o}}(q)$ valid for all $q^{17}:$

$$
\begin{gathered}
S_{\mathrm{aa}}^{\mathrm{o}}=\phi_{\mathrm{a}} N_{\mathrm{a}} P\left(N_{\mathrm{a}}, \alpha_{\mathrm{a}}\right) \\
P\left(N_{\mathrm{a}}, \alpha_{\mathrm{a}}\right)=\frac{1}{N_{\mathrm{a}}}\left\{1+2\left(\mathrm{e}^{\alpha_{\mathrm{a}}}-1\right)^{-1}\left[1-\frac{\left(1-\mathrm{e}^{-N_{\mathrm{a}} \alpha_{\mathrm{a}}}\right)}{N_{\mathrm{a}}\left(1-\mathrm{e}^{-\alpha_{\mathrm{a}}}\right)}\right]\right\} \\
(10 \mathrm{~b}) \\
\alpha_{\mathrm{a}}=\frac{q^{2} a^{2}}{6}
\end{gathered}
$$

where $\phi_{\mathbf{a}}$ is the volume fraction of monomers of type A and $N_{\mathrm{a}}$ is the number of statistical segments. Note that the choice of the normalization used in equation (10a) for the static structure factor is consistent with the normalization of the mobilities in equation (6). In the limit of $a \rightarrow 0$ and $N_{\mathrm{a}} \rightarrow \infty$, keeping $R_{\mathrm{ga}}^{2}=N_{\mathrm{a}} a^{2} / 6$ constant, $P\left(N_{\mathrm{a}}, \alpha_{\mathrm{a}}\right)$ reproduces the conventional Debye form :

$$
P_{\mathrm{D}}\left(x_{\mathrm{a}}\right)=\frac{2}{x_{\mathrm{a}}^{2}}\left(x_{\mathrm{a}}-1+\mathrm{e}^{-x_{\mathrm{a}}}\right)
$$

where $x_{\mathrm{a}}=N_{\mathrm{a}} \alpha_{\mathrm{a}}=\left(q R_{\mathrm{ga}}\right)^{2}$, and $R_{\mathrm{ga}}$ is the radius of gyration of an $A$ chain. The difference between the full expression $P\left(N_{\mathrm{a}}, \alpha_{\mathrm{a}}\right)$ and the Debye form $P_{\mathrm{D}}\left(x_{\mathrm{a}}\right)$ is that the former behaves as $1 / N_{\mathrm{a}}$ in the high $q$ region where $q a \gg 1$, whereas the latter behaves as $2 / x_{\mathrm{a}}$. In the intermediate and high $q$ regions where $q R_{\mathrm{ga}} \gg 1, P\left(N_{\mathrm{a}}, \alpha_{\mathrm{a}}\right)$ simplifies to

$$
P\left(N_{\mathrm{a}}, \alpha_{\mathrm{a}}\right)=\frac{1}{N_{\mathrm{a}}} \operatorname{coth}\left(\frac{\alpha_{\mathrm{a}}}{2}\right)
$$

The static structure factors $S_{\mathrm{bb}}^{\mathbf{o}}(q)$ and $S_{\mathrm{cc}}^{\mathbf{o}}(q)$ are obtained from equation (10) by replacing the properties of $A$ chains by those of $B$ and $C$ chains. The partial structure factors $S_{\mathrm{ac}}^{\mathrm{o}}(q)$ and $S_{\mathrm{bc}}^{\mathrm{o}}(q)$ are zero, because C chains, which are assumed to be homopolymers, are not connected to the A or B chains. The $S_{\mathbf{a b}}^{\mathbf{o}}(q)$ is also zero if $\mathrm{A}$ and $\mathrm{B}$ species are homopolymers, and non-zero when they represent two arms of a copolymer. The calculation of $S_{\mathrm{ab}}^{\mathrm{o}}(q)$ in the latter case is somewhat complicated, though straightforward, when the statistical segment lengths of the two arms are not the same. For diblock copolymer A-B, it can be calculated as

$$
S_{\mathrm{ab}}^{\mathrm{o}}=\sqrt{\phi_{\mathrm{a}} \phi_{\mathrm{b}} N_{\mathrm{a}} N_{\mathrm{b}}} P_{\mathrm{ab}}\left(N_{\mathrm{a}}, N_{\mathrm{b}}, \alpha_{\mathrm{a}}, \alpha_{\mathrm{b}}\right)
$$

where

$$
\begin{aligned}
& P_{\mathrm{ab}}\left(N_{\mathrm{a}}, N_{\mathrm{b}}, \alpha_{\mathrm{a}}, \alpha_{\mathrm{b}}\right) \\
& =\frac{1}{N_{\mathrm{a}} N_{\mathrm{b}}} \mathrm{e}^{-\left(\alpha_{\mathrm{a}}+\alpha_{\mathrm{b}}\right) / 2}\left[\frac{\left(1-\mathrm{e}^{-\alpha_{\mathrm{a}} N_{\mathrm{a}}}\right)\left(1-\mathrm{e}^{-\alpha_{\mathrm{b}} N_{\mathrm{b}}}\right)}{\left(1-\mathrm{e}^{-\alpha_{\mathrm{a}}}\right)\left(1-\mathrm{e}^{-\alpha_{\mathrm{b}}}\right)}\right]
\end{aligned}
$$

In the small and intermediate $q$ regions defined above, equation (13b) simplifies to

$$
P_{\mathrm{ab}}\left(x_{\mathrm{a}}, x_{\mathrm{b}}\right)=\left[\frac{\left(1-\mathrm{e}^{-x_{\mathrm{a}}}\right)\left(1-\mathrm{e}^{-x_{\mathrm{b}}}\right)}{x_{\mathrm{a}} x_{\mathrm{b}}}\right]
$$

whereas in the intermediate and high $q$ regions it becomes

$$
P_{\mathrm{ab}}\left(N_{\mathrm{a}}, N_{\mathrm{b}}, \alpha_{\mathrm{a}}, \alpha_{\mathrm{b}}\right)=\frac{1}{4 \sinh \left(\frac{\alpha_{\mathrm{a}}}{2}\right) \sinh \left(\frac{\alpha_{\mathrm{b}}}{2}\right)}
$$

Finally, when the segment lengths $a$ and $b$ of the A and B chains are the same, i.e. $\alpha_{a}=\alpha_{b}=\alpha$, equation (13b) simplifies to

$$
\begin{aligned}
P_{\mathrm{ab}}\left(N_{\mathrm{a}}, N_{\mathrm{b}}, \alpha\right)= & \frac{1}{2}\left\{\frac{\left(N_{\mathrm{a}}+N_{\mathrm{b}}\right)^{2}}{N_{\mathrm{a}} N_{\mathrm{b}}} P\left[\left(N_{\mathrm{a}}+N_{\mathrm{b}}\right), \alpha\right]\right. \\
& \left.-\frac{N_{\mathrm{a}}}{N_{\mathrm{b}}} P\left(N_{\mathrm{a}}, \alpha\right)-\frac{N_{\mathrm{b}}}{N_{\mathrm{a}}} P\left(N_{\mathrm{b}}, \alpha\right)\right\}
\end{aligned}
$$

In the intermediate and small $q$ regions, $P\left(N_{j}, \alpha\right), j=a$ or $b$, on the right-hand side of equation (16) can be replaced by the Debye form, and the conventional expression of $S_{\mathrm{ab}}^{\mathrm{o}}(q)$ in terms of the Debye forms for the full chain and the two parts of the diblock copolymer is recaptured. Detailed calculation of $P_{\mathrm{ab}}$ for multiblock copolymers having different architecture can be found in reference 18 . We presented the generalized form in equation ( $13 \mathrm{~b}$ ) for the sake of completeness to include the case of copolymers with different segment lengths. In the application of these theoretical results to the interpretation of Stühn and Rennie's experiment, we shall assume that the statistical segment length of polystyrene and polyisoprene in copolymers are the same, and use equation (16) in equation (13a) to calculate $S_{\mathrm{ab}}^{\mathrm{o}}(q)$.

We now have the complete set of equations for the interpretation of dynamic scattering experiments on arbitrary ternary mixtures of homopolymers A and B, or diblock copolymers $\mathbf{A}-\mathbf{B}$, in a matrix of homopolymers $C$. They are simplified considerably under the conditions of the experiments by Stühn and Rennie, in which the ternary system consisted of diblock copolymer of protonated polyisoprene $(A)$ and deuterated polystyrene $(B)$ in the matrix of deuterated polyisoprene (C). Since the scattering lengths of deuterated polyisoprene and deuterated polystyrene are the same, the scattering is only from protonated polyisoprene, which constitutes the labelled component $\mathrm{A}$. Furthermore, the interaction parameters between $\mathrm{B}-\mathrm{C}$ and $\mathrm{B}-\mathrm{A}$ components are taken to be the 
same, assuming that the deuteration does not change the interaction between these species. In this case we have only one $\kappa$ parameter, namely $\kappa=\kappa_{\mathrm{ab}}=\kappa_{\mathrm{bc}}$, and $\kappa_{\mathrm{ac}}=0$, as one can see from equation (8c) with $W_{\mathrm{bc}}=W_{\mathrm{ab}}$, $W_{\text {ac }}=W_{\text {aa }}=W_{\text {cc }}$. With these identifications the static structure factors in the interacting system can be obtained from equation (9) as:

$$
\begin{aligned}
& S_{\mathrm{aa}}(q)=\frac{S_{\mathrm{aa}}^{\mathrm{o}} S_{\mathrm{cc}}^{\mathrm{o}}+\Delta^{\mathrm{o}}-2 \kappa S_{\mathrm{cc}}^{\mathrm{o}} \Delta^{\mathrm{o}}}{S_{\mathrm{aa}}^{\mathrm{o}}+S_{\mathrm{bb}}^{\mathrm{o}}+2 S_{\mathrm{ab}}^{\mathrm{o}}+S_{\mathrm{cc}}^{\mathrm{o}}-2 \kappa\left(S_{\mathrm{bb}}^{\mathrm{o}} S_{\mathrm{cc}}^{\mathrm{o}}+\Delta^{\mathrm{o}}\right)} \\
& S_{\mathrm{bb}}(q)=\frac{S_{\mathrm{bb}}^{\mathrm{o}} S_{\mathrm{cc}}^{\mathrm{o}}+\Delta^{\mathrm{o}}}{S_{\mathrm{aa}}^{\mathrm{o}}+S_{\mathrm{bb}}^{\mathrm{o}}+2 S_{\mathrm{ab}}^{\mathrm{o}}+S_{\mathrm{cc}}^{\mathrm{o}}-2 \kappa\left(S_{\mathrm{bb}}^{\mathrm{o}} S_{\mathrm{cc}}^{\mathrm{o}}+\Delta^{\mathrm{o}}\right)} \\
& S_{\mathrm{ab}}(q)=S_{\mathrm{ba}}(q)=\frac{S_{\mathrm{ab}}^{\mathrm{o}} S_{\mathrm{cc}}^{\mathrm{o}}-\Delta^{\mathrm{o}}}{S_{\mathrm{aa}}^{\mathrm{o}}+S_{\mathrm{bb}}^{\mathrm{o}}+2 S_{\mathrm{ab}}^{\mathrm{o}}+S_{\mathrm{cc}}^{\mathrm{o}}-2 \kappa\left(S_{\mathrm{bb}}^{\mathrm{o}} S_{\mathrm{cc}}^{\mathrm{o}}+\Delta^{\mathrm{o}}\right)}
\end{aligned}
$$

where $\Delta^{\circ}=S_{\mathrm{aa}}^{\mathrm{o}} S_{\mathrm{bb}}^{\mathrm{o}}-S_{\mathrm{ab}}^{\mathrm{o} 2}$, as defined earlier in equation (9d). We remark here that the form of $S_{\mathrm{bb}}(q)$ given in equation (17a) can also be obtained directly from a formula of the static structure factor for diblock copolymers in the melt [equation (32) in ref. 7], which we reproduce here

$$
\begin{aligned}
\frac{1}{S_{\mathrm{AA}}(q)} & =\frac{1}{S_{\mathrm{BB}}(q)}=-\frac{1}{S_{\mathrm{AB}}(q)} \\
& =\mathbf{M}^{\mathrm{T}}\left[\begin{array}{ll}
S_{\mathrm{AA}}^{\mathrm{o}} & S_{\mathrm{AB}}^{\mathrm{o}} \\
S_{\mathrm{BA}}^{\mathrm{o}} & S_{\mathrm{BB}}^{\mathrm{o}}
\end{array}\right]^{-1} \mathbf{M}-2 \kappa
\end{aligned}
$$

where $\mathbf{M}=\operatorname{col}[1,-1]$. When the matrix component is the same species as one of the two species in the copolymers, i.e. $\mathrm{A}=\mathrm{C}$, we can treat all the $\mathrm{A}$ monomers as one component, and regard the ternary system as a binary incompressible mixture of $\mathrm{A}$ and $\mathrm{B}$ with densities $\left(\rho_{\mathrm{a}}+\rho_{\mathrm{c}}\right)$ and $\rho_{\mathrm{b}}$. This procedure is tantamount to identifying in equation (18), $S_{\mathrm{AA}}^{\mathrm{o}}(q)=S_{\mathrm{aa}}^{\mathrm{o}}(q)+S_{\mathrm{cc}}^{\mathrm{o}}(q)$, $S_{\mathrm{AB}}^{\mathrm{o}}(q)=S_{\mathrm{ab}}^{\mathrm{o}}(q)$ and $S_{\mathrm{BB}}^{\mathrm{o}}(q)=S_{\mathrm{bb}}^{\mathrm{o}}(q)$. The $S_{\mathrm{ac}}^{\mathrm{o}}(q)=0$ and $S_{\mathrm{bc}}^{\mathrm{o}}(q)=0$ because there is no connectivity between the matrix homopolymers $\mathrm{A}$ and the $\mathrm{A}$ polymers in the copolymers. One can verify that equation (17b) is indeed obtained from equation (18) with this identification. However, this procedure cannot be used to obtain the expression of $S_{\mathrm{aa}}(q)$ given in equation (17a), because $S_{\mathrm{aa}}(q)$ is the structure factor associated with the A segments only in the copolymers. The reduction procedure described here in the case of a ternary system can also be implemented in mixtures with an arbitrary number of components provided they are all formed by two chemical species (see Appendix).

It seems that the above procedure was used by Stün and Rennie to obtain $S_{\mathrm{aa}}(q)$ and $S_{\mathrm{bb}}(q)$, starting from equation (18). However, the bare structure factors given by equation (10) in this reference reproduces $S_{\mathbf{b b}}(q)$ in the interacting system [equation (1) in ref. 4], which seems to be taken as $S_{\mathrm{aa}}(q)$. Since the first cumulant is being calculated for the labelled A component, this interchange leads to an inconsistent result. In the high $q$ region considered by these authors, however, this difference makes little quantitative difference.

\section{FIRST CUMULANT}

The dynamic scattering experiments are often interpreted in terms of the first cumulant, $\Gamma_{\mathrm{aa}}(q)$, of the observed scattering function $S_{\mathrm{aa}}(q, t)$, rather than using its full expression given in equation (1). The first cumulant is defined by

$$
\Gamma_{\mathrm{aa}}(q)=-\lim _{t \rightarrow 0} \frac{\mathrm{d} S_{\mathrm{aa}}(q, t)}{\mathrm{d} t} \frac{1}{S_{\mathrm{aa}}(q)}
$$

Using equation (1) one obtains

$$
\Gamma_{\mathrm{aa}}(q)=q^{2} k_{\mathrm{B}} T \frac{m_{\mathrm{aa}}}{S_{\mathrm{aa}}(q)}
$$

where $m_{\mathrm{aa}}$ and $S_{\mathrm{aa}}(q)$ are given by equations (5a) and (17a). Since the first cumulant of $S_{\mathrm{aa}}(q, t)$ was measured by Stühn and Rennie ${ }^{4}$ in their spin-echo neutron scattering experiment, we calculate $\Gamma_{\mathrm{aa}}(q)$ explicitly for the mixture of $\mathrm{A}-\mathrm{B}$ copolymers in the matrix of $\mathrm{C}=\mathrm{A}$ homopolymers. Using equation ( $5 \mathrm{a}$ ) for $m_{\mathrm{aa}}$ and equation (17a) for $S_{\mathrm{aa}}(q)$, we find explicitly

$$
\begin{aligned}
\Gamma_{\mathrm{aa}}(q)= & q^{2} k_{\mathrm{B}} T \frac{m_{\mathrm{aa}}^{\mathrm{o}}\left(m_{\mathrm{bb}}^{\mathrm{o}}+m_{\mathrm{cc}}^{\mathrm{o}}\right)}{\left(m_{\mathrm{aa}}^{\mathrm{o}}+m_{\mathrm{bb}}^{\mathrm{o}}+m_{\mathrm{cc}}^{\circ}\right)} \\
& \times \frac{S_{\mathrm{aa}}^{\mathrm{o}}+S_{\mathrm{bb}}^{\mathrm{o}}+2 S_{\mathrm{ab}}^{\mathrm{o}}+S_{\mathrm{cc}}^{\mathrm{o}}-2 \kappa\left(S_{\mathrm{bb}}^{\mathrm{o}} S_{\mathrm{cc}}^{\mathrm{o}}+\Delta^{\circ}\right)}{\left(S_{\mathrm{aa}}^{\mathrm{o}} S_{\mathrm{cc}}^{\mathrm{o}}+\Delta^{\circ}-2 \kappa S_{\mathrm{cc}}^{\mathrm{o}} \Delta^{\circ}\right)}
\end{aligned}
$$

where, now $m_{\mathrm{cc}}^{\mathrm{o}}=(1-\phi) / \zeta_{\mathrm{a}}$ and

$$
S_{\mathrm{cc}}^{\mathrm{o}}(q)=(1-\phi) N_{\mathrm{c}} P\left(N_{\mathrm{c}}, \alpha_{\mathrm{c}}\right)
$$

If the B monomers in the copolymers were labelled, the first cumulant of scattering intensity, $S_{b b}(q, t)$, would be written by using equations $(17 \mathrm{~b})$ and $(5 \mathrm{~b})$ as follows:

$$
\begin{aligned}
\Gamma_{\mathrm{bb}}(q)= & q^{2} k_{\mathrm{B}} T \frac{m_{\mathrm{bb}}^{\mathrm{o}}\left(m_{\mathrm{aa}}^{\mathrm{o}}+m_{\mathrm{cc}}^{\mathrm{o}}\right)}{\left(m_{\mathrm{aa}}^{\mathrm{o}}+m_{\mathrm{bb}}^{\mathrm{o}}+m_{\mathrm{cc}}^{\mathrm{o}}\right)} \\
& \times \frac{S_{\mathrm{aa}}^{\mathrm{o}}+S_{\mathrm{bb}}^{\mathrm{o}}+2 S_{\mathrm{ab}}^{\mathrm{o}}+S_{\mathrm{cc}}^{\mathrm{o}}-2 \kappa\left(S_{\mathrm{bb}}^{\mathrm{o}} S_{\mathrm{cc}}^{\mathrm{o}}+\Delta^{\mathrm{o}}\right)}{\left(S_{\mathrm{bb}}^{\mathrm{o}} S_{\mathrm{cc}}^{\mathrm{o}}+\Delta^{\mathrm{o}}\right)}
\end{aligned}
$$

We included this case for completeness, as well as for future discussions, even though we only need equation (21a) to interpret the experimental results by Stühn and Rennie $^{4}$. Both equations are valid for all values of $q$, including the intermediate and high $q$ regions, where Stühn and Rennie's experiment was performed. Since the objective of this paper is broader than just interpreting this particular experiment, we shall digress here to study the variation of $\Gamma_{\mathrm{aa}}(q)$ and $\Gamma_{\mathrm{bb}}(q)$ with $q$ in all $q$ regions, and investigate the influence of the interaction parameter on its behaviour.

\section{SMALL $q$ REGION : $q R_{\mathrm{g}} \ll 1$}

In this $q$ region, the bare system static structure factors can be approximated by

$$
\begin{aligned}
& S_{\mathrm{aa}}^{\mathrm{o}}(q)=\phi N f_{\mathrm{a}}^{2}\left(1-q^{2} R_{\mathrm{ga}}^{2} / 3\right) \\
& S_{\mathrm{bb}}^{\mathrm{o}}(q)=\phi N\left(1-f_{\mathrm{a}}\right)^{2}\left(1-q^{2} R_{\mathrm{gb}}^{2} / 3\right) \\
& S_{\mathrm{ab}}^{\mathrm{o}}(q)=\phi N f_{\mathrm{a}}\left(1-f_{\mathrm{a}}\right)\left[1-q^{2}\left(R_{\mathrm{ga}}^{2}+R_{\mathrm{gb}}^{2}\right) / 2\right](22 \mathrm{c}) \\
& S_{\mathrm{cc}}^{\mathrm{o}}(q)=(1-\phi) N_{\mathrm{c}}\left(1-q^{2} R_{\mathrm{gc}}^{2} / 3\right)
\end{aligned}
$$

Substitution of these expressions into the expression of $S_{\mathrm{aa}}(q)$ given in equation (17a) and ignoring terms of the order of $q^{4}$ or higher, one obtains the expression for 
$S_{\text {aa }}(q)$ in this $q$ range:

$$
S_{\mathrm{aa}}(q)=S_{\mathrm{aa}}(0) /\left(1+q^{2} \xi_{\mathrm{c}}^{2}\right)
$$

where the correlation length $\xi_{\mathrm{c}}$ is found to be

$$
\begin{aligned}
& \xi_{\mathrm{c}}=\frac{a}{6\left(1-f_{\mathrm{a}}\right)\left(\kappa_{\mathrm{c}}^{\text {macro }}-\kappa\right)^{1 / 2}} \\
& \times\left\{\begin{array}{l}
\frac{f_{\mathrm{c}}+\left(1-f_{\mathrm{a}}\right)\left(2 f_{\mathrm{a}}-3\right)}{f_{\mathrm{c}}(1-\phi)}+\frac{f_{\mathrm{a}}}{\phi}-\frac{2 \phi\left(1-f_{\mathrm{a}}\right)^{2}}{(1-\phi)^{2} f_{\mathrm{c}}^{2}} \\
+2 \kappa N\left(1-f_{\mathrm{a}}\right)^{2}\left[\frac{4 \phi\left(1-f_{\mathrm{a}}\right)}{(1-\phi) f_{\mathrm{c}}}+f_{\mathrm{c}}+4+f_{\mathrm{a}}\right]-8 \phi\left[\kappa N\left(1-f_{\mathrm{a}}\right)\right]^{2}
\end{array}\right\}^{1 / 2}
\end{aligned}
$$

where $f_{c}=N_{\mathrm{c}} / N$ denotes the size of the A homopolymer relative to the size of the copolymers and $f_{\mathrm{a}}=N_{\mathrm{a}} / N$ where $N$ is the total number of segments in a copolymer molecule. The term $\kappa_{c}^{\text {macro }}$ denotes the critical value of the $\kappa$ parameter where the macrophase separation sets in, and is defined as

$$
\mathcal{K}_{\mathrm{c}}^{\text {macro }}=\frac{1}{2\left(1-f_{\mathrm{a}}\right)^{2}}\left[\frac{1}{\phi N}+\frac{1}{(1-\phi) N_{\mathrm{c}}}\right]
$$

Since the bare system mobilities in equation (21a) are independent of $q$, the small $q$ limit of $\Gamma_{\mathrm{aa}}(q)$ can be written as

$$
\begin{aligned}
\Gamma_{\mathrm{aa}}(q)= & 2 q^{2} k_{\mathrm{B}} T \frac{\phi f_{\mathrm{a}}\left(1-\phi f_{\mathrm{a}}\right)}{\bar{\zeta}_{\mathrm{a}}}\left(\frac{1-f_{\mathrm{a}}}{f_{\mathrm{a}}}\right)^{2} \\
& \times\left(\kappa_{\mathrm{c}}^{\text {macro }}-\kappa\right)\left(1+q^{2} \xi_{\mathrm{c}}^{2}\right)
\end{aligned}
$$

where $\bar{\zeta}_{\mathbf{a}}$ is an effective friction coefficient defined as

$$
\frac{1}{\bar{\zeta}_{\mathrm{a}}}=\frac{1}{\zeta_{\mathrm{a}}} \frac{1}{\left(1-\phi_{\mathrm{a}}\right)} \frac{\phi_{\mathrm{c}} \zeta_{\mathrm{b}}+\phi_{\mathrm{b}} \zeta_{\mathrm{a}}}{\left(1-\phi_{\mathrm{b}}\right) \zeta_{\mathrm{b}}+\phi_{\mathrm{b}} \zeta_{\mathrm{a}}}
$$

where $\phi_{\mathrm{a}}=\phi f_{\mathrm{a}}, \phi_{\mathrm{b}}=\phi\left(1-f_{\mathrm{a}}\right)$ and $\phi_{\mathrm{c}}=1-\phi$. The small $q$ limit of $\Gamma_{\mathrm{aa}}(q) / q^{2}$ defines the short time diffusion coefficient of the A chains, which follows from equation $(25)$ as

$$
D_{\mathrm{sh}}=2 k_{\mathrm{B}} T \frac{\phi f_{\mathrm{a}}\left(1-\phi f_{\mathrm{a}}\right)}{\bar{\zeta}_{\mathrm{a}}}\left(\frac{1-f_{\mathrm{a}}}{f_{\mathrm{a}}}\right)^{2}\left(\kappa_{\mathrm{c}}^{\text {macro }}-\kappa\right)
$$

It is interesting to demonstrate how the above general results for a ternary mixture reproduce, as special cases, some known results in binary mixtures. The limit of $f_{\mathrm{a}} \rightarrow 1$ corresponds to an incompressible binary mixture of A molecules with different molecular weights, because $f_{\mathrm{a}}=1$ implies that the B component in diblock copolymers is absent. Since the remaining components consist of molecules of the same species, the $\kappa$ parameter is zero, assuming that the protonated and deuterated species do not interact. Therefore equation (25) reduces to

$$
\Gamma_{\mathrm{aa}}(q)=\frac{q^{2} k_{\mathrm{B}} T}{\zeta_{\mathrm{a}}}\left[\frac{(1-\phi)}{N_{\mathrm{a}}}+\frac{\phi}{N_{\mathrm{c}}}\right]\left[1+q^{2} \xi_{\mathrm{c}}^{2}\right]
$$

where the correlation length $\xi_{\mathrm{c}}$ is simplified as

$$
\xi_{\mathrm{c}}^{2}=\frac{a^{2}}{18}\left[\frac{(1-\phi)}{N_{\mathrm{a}}}+\frac{\phi}{N_{\mathrm{c}}}\right]^{-1}
$$

The short time diffusion coefficient in equation (27) in this particular mixture becomes

$$
D_{\mathrm{sh}}=\frac{k_{\mathrm{B}} T}{\zeta_{\mathrm{a}}}\left[\frac{(1-\phi)}{N_{\mathrm{a}}}+\frac{\phi}{N_{\mathrm{c}}}\right]
$$

which is the interdiffusion coefficient for this binary system consisting of molecules of the same species with different molecular weights. When $\phi \rightarrow 0($ or $\phi \rightarrow 1)$, equation (29) reduces to the self diffusion coefficient of an A molecule, i.e. $D_{\mathrm{s}}=k_{\mathrm{B}} T / \zeta_{\mathrm{a}} N_{\mathrm{a}}$.

If we let $f_{\mathrm{a}} \rightarrow 0$ in equation ( $21 \mathrm{~b}$ ), we obtain the first cumulant in an interacting binary mixture of homopolymers A and B discussed by de Gennes ${ }^{19}$, Binder $^{14}$ and Akcasu et al.?

$$
\Gamma_{\mathrm{bb}}(q)=2 \frac{q^{2} k_{\mathrm{B}} T}{\bar{\zeta}}\left(\kappa_{\mathrm{c}}^{\text {macro }}-\kappa\right)\left(1+q^{2} \xi_{\mathrm{c}}^{2}\right)
$$

where

$$
\begin{aligned}
\xi_{c}^{2} & =\frac{a^{2}}{36} \frac{1}{\phi(1-\phi)\left(\kappa_{\mathrm{c}}^{\text {macro }}-\kappa\right)} \\
\bar{\zeta} & =(1-\phi) \zeta_{\mathrm{b}}+\phi \zeta_{\mathrm{a}}
\end{aligned}
$$

and $\kappa_{\mathrm{c}}^{\text {macro }}$ is given by equation (24) with $f_{\mathrm{a}}=0$. Of course, the results of the limit of $f_{\mathrm{a}} \rightarrow 1$ discussed above are obtained from these equations by letting $\zeta_{\mathbf{a}}=\zeta_{\mathbf{b}}$ and $\kappa=0$. The short time diffusion coefficient for this particular case is obtained as

$$
D_{\mathrm{sh}}=\frac{2 k_{\mathrm{B}} T}{\bar{\zeta}}\left(\kappa_{\mathrm{c}}^{\text {macro }}-\kappa\right)
$$

which corresponds to the interdiffusion coefficient in an interacting incompressible binary homopolymer mixture.

Another limiting case, which corresponds to a copolymer melt, is obtained by taking the limit of $\phi \rightarrow 1$. The resulting equations reproduce those obtained by Akcasu et $\mathrm{al}^{7}$ directly in copolymer melts. The first cumulant follows from equation (21a) or equation (21b) as

$$
\begin{gathered}
\Gamma_{\mathrm{aa}}(q)=q^{2} k_{\mathrm{B}} T \frac{f_{\mathrm{a}}\left(1-f_{\mathrm{a}}\right)}{\bar{\zeta}}\left[\frac{6}{N q^{2}\left(R_{\mathrm{ga}}^{2}+R_{\mathrm{gb}}^{2}\right)}-2 k\right] \\
\bar{\zeta}=\left(1-f_{\mathrm{a}}\right) \zeta_{\mathrm{a}}+f_{\mathrm{a}} \zeta_{\mathrm{b}}
\end{gathered}
$$

One can verify that $\Gamma_{\mathrm{aa}}(q)=\Gamma_{\mathrm{bb}}(q)$. It is observed that $\Gamma_{\mathrm{aa}}(q)$ does not vanish as $q \rightarrow 0$, but rather tends to a finite relaxation frequency, indicating that initial relaxation of long wave density inhomogeneities is due to local motions of the two arms of the copolymer, and becomes independent of $q$ whenever $q R_{\mathrm{g}} \ll 1$ (ref. 7 ). In equation (32a), the first term in parentheses is always much larger than the second term $2 \kappa N$ in the small $q$ region. It is kept only to show that the interactions tend to decrease the relaxation frequency. Indeed, $\Gamma_{\mathrm{aa}}(q)$ vanishes when $\kappa$ becomes equal to $\kappa_{\mathrm{c}}^{\text {micro }}$, at the critical value $q_{\mathrm{c}}$, which corresponds to the beginning of the microphase separation. Since $q_{\mathrm{c}} R_{\mathrm{gT}} \sim 2$, equation (32a) is not applicable in this $q$ range. For $\kappa<\kappa_{\mathrm{c}}^{\text {micro }}, \Gamma_{\mathrm{aa}}(q)$ passes through a minimum, and begins to increase for larger values of $q$ towards the intermediate $q$ values. Its behaviour in this asymptotic $q$ region is discussed below.

\section{INTERMEDIATE $q$ REGION $: q a \ll 1, q R_{\mathrm{g}} \gg 1$}

In this $q$ region, the bare system static structure factors can be approximated by

$$
\begin{aligned}
& S_{\mathrm{aa}}^{\mathrm{o}}(q)=2 \phi N f_{\mathrm{a}}^{2} / q^{2} R_{\mathrm{ga}}^{2} \\
& S_{\mathrm{bb}}^{\mathrm{o}}(q)=2 \phi N\left(1-f_{\mathrm{a}}\right)^{2} / q^{2} R_{\mathrm{gb}}^{2} \\
& S_{\mathrm{ab}}^{\mathrm{o}}(q)=\phi N f_{\mathrm{a}}\left(1-f_{\mathrm{a}}\right) / q^{4} R_{\mathrm{ga}}^{2} R_{\mathrm{gb}}^{2}
\end{aligned}
$$




$$
S_{\mathrm{cc}}^{\mathrm{o}}(q)=2(1-\phi) N_{\mathrm{c}} / q^{2} R_{\mathrm{gc}}^{2}
$$

The first cumulant $\Gamma_{\mathrm{aa}}(q)$ in the intermediate $q$ region can be written as

$$
\begin{aligned}
& \Gamma_{\mathrm{aa}}(q)=\frac{k_{\mathrm{B}} T}{\bar{\zeta}_{\mathrm{a}}} \frac{q^{4} a^{2}}{12} \times \\
& \left\{1+\phi\left(1-f_{\mathrm{a}}\right)\left(\frac{a^{2}}{b^{2}}-1\right)\right. \\
& \left.\quad-2 \kappa\left\{\frac{12}{q^{2} b^{2}}\left[\phi(1-\phi)\left(1-f_{\mathrm{a}}\right)+\phi^{2} f_{\mathrm{a}}\left(1-f_{\mathrm{a}}\right)\right]\right\}\right\} \\
& \frac{(1-\phi)}{\left(1-\phi f_{\mathrm{a}}\right)}+\frac{\phi\left(1-f_{\mathrm{a}}\right)}{\left(1-\phi f_{\mathrm{a}}\right)} \frac{a^{2}}{b^{2}}-2 \kappa\left[\frac{12 \phi(1-\phi)\left(1-f_{\mathrm{a}}\right)}{q^{2} b^{2}\left(1-\phi f_{\mathrm{a}}\right)}\right]
\end{aligned}
$$

When segment lengths of $A$ and $B$ polymers are equal to each other, equation (34a) simplifies to

$$
\begin{aligned}
& \Gamma_{\mathrm{aa}}(q)=\frac{k_{\mathrm{B}} T}{\bar{\zeta}_{\mathrm{a}}} \frac{q^{4} a^{2}}{12} \\
& \times \frac{1-2 \kappa\left\{\frac{12}{q^{2} a^{2}}\left[\phi(1-\phi)\left(1-f_{\mathrm{a}}\right)+\phi^{2} f_{\mathrm{a}}\left(1-f_{\mathrm{a}}\right)\right]\right\}}{1-2 \kappa\left[\frac{12}{q^{2} a^{2}} \frac{\phi(1-\phi)\left(1-f_{\mathrm{a}}\right)}{1-\phi f_{\mathrm{a}}}\right]}
\end{aligned}
$$

where $\bar{\zeta}_{\mathrm{a}}$ is given in equation (26). The usual $q^{4}$ behaviour of the first cumulant in the intermediate $q$ region, which is a characteristic of Rouse dynamics, is recaptured in equation (34) with an additional factor, involving the interaction parameter $\kappa$. When the $\mathrm{B}$ monomers are labelled, one measures the first cumulant $\Gamma_{\mathrm{bb}}(q)$ of $S_{\mathrm{bb}}(q, t)$. The asymptotic behaviour of $\Gamma_{\mathrm{bb}}(q)$ follows from equation $(21 \mathrm{~b})$ as

$$
\begin{aligned}
\Gamma_{\mathrm{bb}}(q)= & \frac{k_{\mathrm{B}} T}{\bar{\zeta}_{\mathrm{b}}} \frac{q^{4} a^{2}}{12} \\
& \times\left\{1-\frac{4 \kappa N_{\mathrm{a}}}{q^{2} R_{\mathrm{ga}}^{2}}\left[\phi(1-\phi)+\phi^{2} f_{\mathrm{a}}\right]\left(1-f_{\mathrm{a}}\right)\right\}
\end{aligned}
$$

where $\bar{\zeta}_{\mathrm{b}}$ is the effective friction coefficient for B monomers and defined by

$$
\bar{\zeta}_{\mathrm{b}}=\left(1-\phi_{\mathrm{b}}\right) \zeta_{\mathrm{b}}+\phi_{\mathrm{b}} \zeta_{\mathrm{a}}
$$

where $\phi_{\mathrm{b}}=\phi\left(1-f_{\mathrm{a}}\right)$. The cases of binary homopolymer blends and copolymer melts in the intermediate $q$ region are obtained as follows. In the limiting case, $f_{\mathrm{a}} \rightarrow 1$, one obtains the incompressible mixture of A-type homopolymers with different molecular weights :

$$
\Gamma_{\mathrm{aa}}(q)=q^{2} \frac{k_{\mathrm{B}} T}{\zeta_{\mathrm{a}}}\left(\frac{q^{2} a^{2}}{12}\right)
$$

Since the motion of the subchains with end-to-end distances much smaller than the full chain size, and larger than the segmental length, are probed in the intermediate $q$ region, all the A chains with different molecular weights have identical first cumulants. Therefore, equation (37) is identical to the first cumulant of a single chain in Rouse dynamics. If we let $f_{\mathrm{a}} \rightarrow 0$ in equation (35), we obtain the first cumulant in an interacting binary mixture of homopolymers $\mathrm{C}=\mathrm{A}$ and $\mathrm{B}$ in the intermediate $q$ region as

$$
\Gamma_{\mathrm{bb}}(q)=\frac{k_{\mathrm{B}} T}{\bar{\zeta}_{\mathrm{b}}} \frac{q^{4} a^{2}}{12}\left[1-\frac{4 \kappa N}{q^{2} R_{\mathrm{gT}}^{2}} \phi(1-\phi)\right]
$$

where $N=N_{\mathrm{b}}+N_{\mathrm{c}}$ and $R_{\mathrm{gT}}^{2}=R_{\mathrm{gb}}^{2}+R_{\mathrm{gc}}^{2}$. Both in equations (35) and (38a), the second terms in parentheses are smaller than unity in the intermediate $q$ region because $\kappa N$ has to be less than its critical value in the one phase region and $q^{2} R_{\mathrm{gT}}^{2} \gg 1$. To clarify this point in a simpler case we rewrite equation (38a) for a symmetric binary blend of B and C homopolymers with $N_{\mathrm{c}}=N_{\mathrm{b}}=$ $N / 2$, by eliminating $\phi(1-\phi)$ in favour of $\kappa_{\mathrm{c}}^{\text {macro }}$, as

$$
\Gamma_{\mathrm{bb}}(q)=\frac{k_{\mathrm{B}} T}{\bar{\zeta}_{\mathrm{b}}} \frac{q^{4} R_{\mathrm{g}}^{2}}{2 N}\left(1-\frac{\kappa}{\kappa_{\mathrm{c}}^{\text {macro }}} \frac{4}{q^{2} R_{\mathrm{gT}}^{2}}\right)
$$

where $\kappa_{\mathrm{c}}^{\text {macro }}$ is defined in equation (24). Since, in the one phase, $\kappa / \kappa_{\mathrm{c}}^{\text {macro }}<1$, the second term is smaller than unity in the intermediate $q$ region. We kept this term only to show the trend of the influence of the interactions on the first cumulant. The limit of $\phi \rightarrow 1$ corresponds to the melt of copolymer A-B. From equations (34b) and (35) we obtain:

$$
\begin{aligned}
\Gamma_{\mathrm{aa}}(q)=\frac{k_{\mathrm{B}} T}{\bar{\zeta}} \frac{q^{4} R_{\mathrm{gT}}^{2}}{2 N} & \\
\times & {\left[1-\frac{4 \kappa N}{q^{2} R_{\mathrm{gT}}^{2}} f_{\mathrm{a}}\left(1-f_{\mathrm{a}}\right)\right] }
\end{aligned}
$$

where $N=N_{\mathrm{b}}+N_{\mathrm{a}}, R_{\mathrm{gT}}^{2}=R_{\mathrm{gb}}^{2}+R_{\mathrm{ga}}^{2}$ and $\bar{\zeta}$ is given by equation (32b). In both equations (38b) and (39), the $q^{4}$ dependence prevails.

\section{LARGE $q$ REGION : $q a \gg 1$}

In order to obtain the large $q$ behaviour of the first cumulant, where the segmental motion is dominant, one has to use the full expression of the static structure factors, as we pointed out earlier. For $q a \gg 1$, the bare system static structure factors can be approximated by

$$
\begin{aligned}
& S_{\mathrm{aa}}^{\mathrm{o}}(q)=\phi f_{\mathrm{a}} \\
& S_{\mathrm{bb}}^{\mathrm{o}}(q)=\phi\left(1-f_{\mathrm{a}}\right) \\
& S_{\mathrm{ab}}^{\mathrm{o}}(q)=0 \\
& S_{\mathrm{cc}}^{\mathrm{o}}(q)=(1-\phi)
\end{aligned}
$$

Hence, the first cumulant in this $q$ region is found from equation (21a) as

$$
\begin{aligned}
\Gamma_{\mathrm{aa}}(q)= & \frac{q^{2} k_{\mathrm{B}} T}{\bar{\zeta}_{\mathrm{a}}} \\
& \times \frac{1-2 \kappa\left[\phi(1-\phi)\left(1-f_{\mathrm{a}}\right)+\phi^{2} f_{\mathrm{a}}\left(1-f_{\mathrm{a}}\right)\right]}{1-2 \kappa\left[\frac{\phi(1-\phi)\left(1-f_{\mathrm{a}}\right)}{\left(1-\phi f_{\mathrm{a}}\right)}\right]}
\end{aligned}
$$

which corresponds to segmental diffusion. From this result, we again obtain the case of a binary mixture of homopolymers of kind A, with two different molecular weights, by taking the limit as $f_{\mathrm{a}} \rightarrow 1$ :

$$
\Gamma_{\mathrm{aa}}(q)=q^{2} \frac{k_{\mathrm{B}} T}{\zeta_{\mathrm{a}}}
$$

The case of copolymer melts follow from equation (41) 
with $\phi \rightarrow 1$ as

$$
\Gamma_{\mathrm{aa}}(q)=q^{2} \frac{k_{\mathrm{B}} T}{\bar{\zeta}}
$$

where $\bar{\zeta}$ is given by equation (32b). Since in this case, the diffusion of two different species is being observed, the friction coefficient in equation (43) is a weighted average of the individual friction coefficients $\zeta_{a}$ and $\zeta_{b}$ as $\left(1-f_{\mathrm{a}}\right) \zeta_{\mathrm{a}}+f_{\mathrm{a}} \zeta_{\mathrm{b}}$. It is to be noted that this superposition of the friction coefficients is consistent with the inverse superposition rule of mobilities given in equation (5). The homopolymer case given in equation (42) is obtained from equation (43) with $\zeta_{a}=\zeta_{b}$.

\section{INTERPRETATION OF STÜHN AND RENNIE'S EXPERIMENT}

The dynamic RPA predicts the variation of the first cumulant of the dynamic scattering function of a labelled component in a multicomponent polymer blend as a function of $q, \kappa$, volume fractions of components and scattering lengths of species. By comparing the theoretical expression of the first cumulant with the experimental data, one can infer the numerical values of various physical parameters, such as the segment lengths, Flory interaction parameters, friction coefficients and number of segments in chains. Since the number of physical parameters to be inferred from the data is large, both static and dynamic scattering experiments on the same system are desirable. From static scattering experiments $^{20,21}$ one can extract information about the Flory interaction parameter and radius of gyration of polymer molecules. Interpretation of dynamic scattering experiments in the high $q$ region in terms of the first cumulant, on the other hand, yields the segment lengths and effective friction coefficients. As an example, we propose to interpret Stühn and Rennie's experimental data we described earlier. In this experiment, the first cumulant in the high $q$ region was measured by quasi elastic neutron

Table 1 Properties of polymer species

\begin{tabular}{llll}
\hline & $M_{\mathrm{w}}$ & $M_{\mathrm{w}} / M_{\mathrm{n}}$ & \multicolumn{1}{c}{$f_{\mathrm{a}}$} \\
\hline p-PI/d-PS & 32400 & 1.04 & 0.5 \\
d-PI & 80000 & 1.03 & \\
& & & $R_{\mathrm{g}}(\mathrm{nm})^{b}$ \\
p-PI & $C(\mathrm{~nm})^{a}$ & & $\sim 3.8$ \\
d-PS & 0.0348 & & $\sim 4.0$ \\
d-PI & 0.0286 & & $\sim 9.7$ \\
\end{tabular}

${ }^{a}$ Data obtained from reference 24

${ }^{b} R_{\mathrm{g}}=C\left(M_{\mathrm{N}}\right)^{1 / 2}$

Table 2 Measured values of $\Gamma_{\mathbf{a a}}(q)$ for different $q$ values and values of $\kappa$

\begin{tabular}{llll}
\hline & $\begin{array}{l}T=438 \mathrm{~K} \\
\Gamma_{\mathrm{aa}}(q)\left(\times 10^{-6}\right) \\
\left(\mathrm{s}^{-1}\right)\end{array}$ & $\begin{array}{l}T=453 \mathrm{~K} \\
\Gamma_{\mathrm{aa}}(q)\left(\times 10^{-6}\right) \\
\left(\mathrm{s}^{-1}\right)\end{array}$ & $\begin{array}{l}T=473 \mathrm{~K} \\
\Gamma_{\mathrm{aa}}(q)\left(\times 10^{-6}\right) \\
\left(\mathrm{s}^{-1}\right)\end{array}$ \\
\hline 0.74 & - & - & $5.9(1.4)$ \\
0.87 & $1.9(0.9)$ & $6.2(1.3)$ & $15.8(1.7)$ \\
0.99 & $8.1(1.5)$ & $14.4(1.7)$ & $29.8(2.1)$ \\
1.24 & $25.1(3.0)$ & $40.9(3.3)$ & $96.1(10.5)$ \\
1.48 & $41.1(6.9)$ & $60.0(6.6)$ & $178.1(16.0)$ \\
$\kappa$ & 0.065 & 0.055 & 0.045 \\
\hline
\end{tabular}

Table 3 Effective friction coefficients $\left(\bar{\zeta}_{\mathrm{a}}\right)^{a}$ and segment lengths $(a)$ at various temperatures

\begin{tabular}{llll}
\hline$T(\mathrm{~K})$ & $10^{-6 \bar{\zeta}} / a^{2}\left(\mathrm{~g} \mathrm{~cm}^{-2} \mathrm{~s}^{-1}\right)$ & $10^{7} \bar{\zeta}_{\mathrm{a}}\left(\mathrm{g} \mathrm{s}^{-1}\right)$ & $a(\mathrm{~nm})$ \\
\hline 438 & 6.6 & 2.57 & 1.81 \\
453 & 3.8 & 1.48 & 2.52 \\
473 & 1.9 & 0.74 & 0.51 \\
\hline
\end{tabular}

${ }^{a}$ Friction coefficients calculated using $a=1.6 \mathrm{~nm}$

scattering at three temperatures and five different scattering angles, far from the spinodal line. In Table 1, we reproduce the molecular weight and the radius of gyration of the styrene-isoprene diblock copolymers and the deuterated polyisoprene used. In Table 2, we present the measured values of the first cumulant $\Gamma_{\mathrm{aa}}(q)$ of the dynamic scattering function $S_{\mathrm{aa}}(q, t)$ of the protonated polyisoprene for five different $q$ values and $\kappa$ values at three different temperatures. The $\kappa$ value is obtained from Figure 3 of reference 4 .

In order to analyse the experimental data, we use equation (21a) with the full description of bare system static structure factors valid for all values of $q$. Since the radius of gyration of the chains and the volume fraction of the components are already specified, the only remaining unknowns in equation (21a) are the statistical segment length of the chains, which we assume to be the same for polyisoprene and polystyrene, and the effective friction coefficient. The Levenberg-Marquart ${ }^{22,23}$ nonlinear curve fitting technique is used to calculate these two parameters by fitting equation (21a) to the data reproduced in Table 2 . The experimental points in the region of segmental diffusion, do not quite level off, and have larger standard deviations compared to those in the intermediate $q$ region. Therefore, the curve fitting algorithm with weighting of the data with the error bars given in Table 2 has yielded only the ratios $\bar{\zeta}_{\mathrm{a}} / a^{2}$ unambiguously at all three temperatures, but yielded unreliable values (too small) for segment length. This is understandable because when the data is weighted with the error bars, the information about the segmental diffusion contained in the last two data points is virtually lost. In order to obtain the segment length, we therefore used data with the same importance, i.e. without weighting with the error bars. The curve fitting produced an averaged segment length $a=1.6(0.8) \mathrm{nm}$ (Table 3). The effective friction coefficients $\bar{\zeta}_{a}$ were obtained by using the same segment length $(a=1.6 \mathrm{~nm})$ at the three temperatures. In Figures $l a$ and $b$, we present the theoretically calculated first cumulant $\Gamma_{\mathrm{aa}}(q)$ as a function of $q$ (solid lines) and the experimental data (symbols) to give an idea about the quality of the agreement between experimental data and theoretical results with weighting of the data with experimental error bars (Figure la) and without weighting (Figure $I b$ ). To obtain the segment length and effective friction coefficient more accurately, one needs more data points with better accuracy in the segmental diffusion region. The segment length $[a=1.6(0.8) \mathrm{nm}]$ we have obtained by using only four data points is still reasonable, particularly considering the fact that we had to assume the segment lengths of polystyrene and polyisoprene to be the same. These results demonstrate at least the potential of spin-echo neutron scattering experiments to measure the statistical segment length and the effective friction coefficients of chains. 

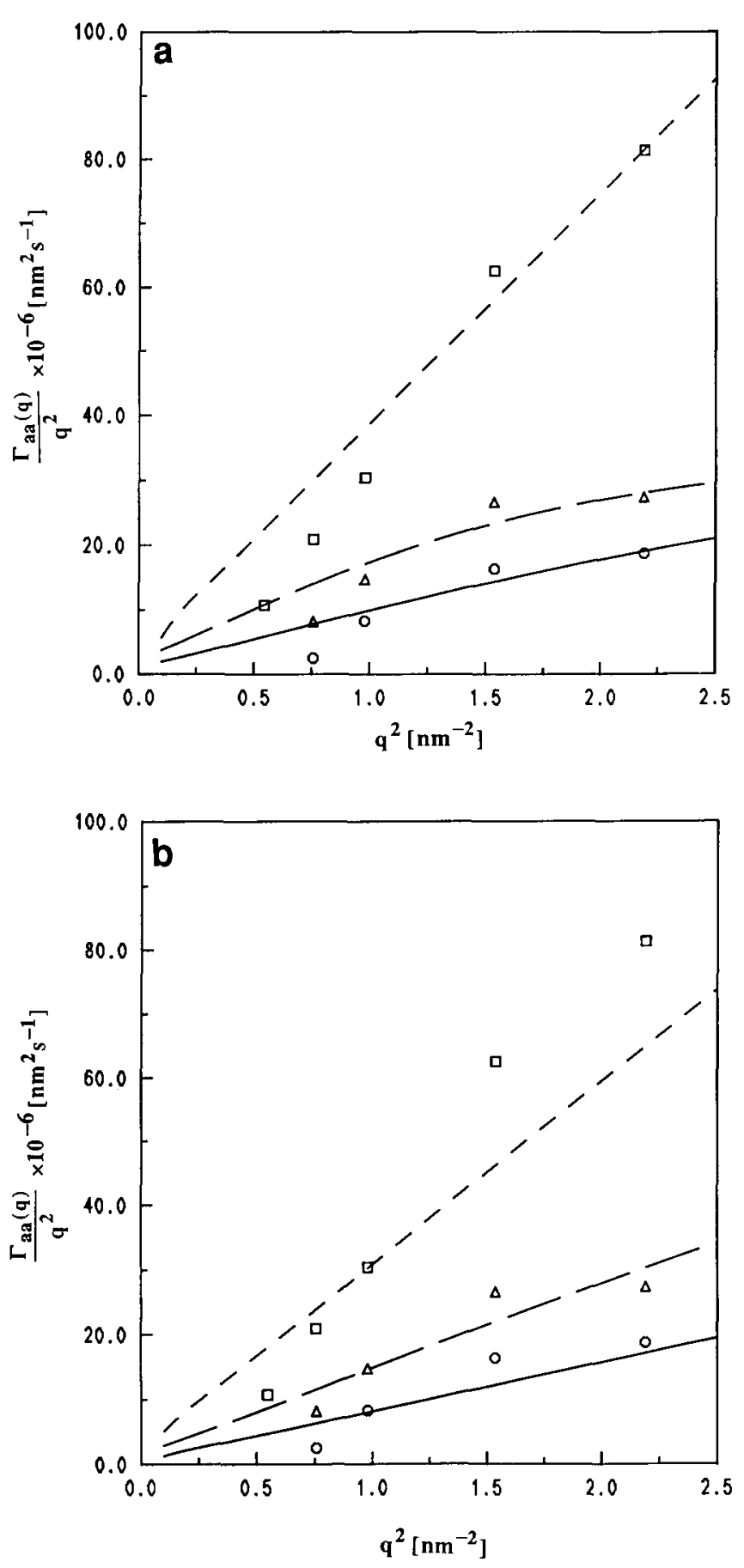

Figure 1 Variation of reduced first cumulant $\Gamma_{\mathrm{aa}}(q) / q^{2}$ as a function of wave number $q$ at three temperatures for the system of diblock copolymers $\mathrm{p}$-PI/d-PS in the matrix of homopolymers d-PI described in Table 1. The symbols show the experimental data and lines show the theoretical fit [equation (21a)], with weighting of the data (a) with the experimental error bars given in Table 2 and without weighting (b). Temperature (K): (-) 438; (- $453 ;(---) 473$; (O) 438; ( $\triangle$ ) $453 ;(\square) 473$

In Figure 2, we plotted the variation of the first cumulant $\Gamma_{\mathrm{aa}}(q)$ as a function of $q$ over the entire $q$ region. As one can see from the graph, the behaviour of the first cumulant depends on the volume fraction of the copolymers and $\kappa$. In general, for a small volume fraction of copolymers, less than the fraction $\phi_{\mathrm{c}}$, which we define presently, we observe the typical behaviour for homopolymer mixtures, in which the relaxation frequency $\Gamma_{\text {aa }}(q)$ decreases with increasing $\kappa$ at $q=0$. For large volume fraction of copolymers $\left(\phi>\phi_{\mathrm{c}}\right)$, we observe that $\Gamma_{\mathrm{aa}}(q)$ tends to a minimum at $q_{\mathrm{c}}$ (also to be defined below ), rather than at $q=0$. This behaviour is characteristic of copolymer melts as discussed previously by Akcasu et al. ${ }^{7}$. For larger values of $q>q_{c}, \Gamma_{\mathrm{aa}}(q)$ displays, at both volume fractions, first a $q^{4}$ power law behaviour in the intermediate $q$ region, and then tends into the high $q$ region, where the segmental diffusion dominates the relaxation of the dynamic scattering function. The various asymptotic regions discussed analytically earlier, and the non-asymptotic transition regions between them, are clearly discernable in Figure 2 .

In order to better understand the observed behaviour of the first cumulant, as well as the variation of the relaxation frequencies and amplitudes of the modes with $q$, we must first digress and discuss the phase diagram of ternary polymer mixtures consisting of $\mathrm{A}-\mathrm{B}$ copolymers and $\mathrm{A}$ homopolymers.

\section{STABILITY CRITERIA FOR PHASE SEPARATION}

It has been shown that ${ }^{24}$ the mechanism and kinetics of phase transition from the disordered to the ordered state in the case of a ternary mixture of copolymers $\mathrm{A}-\mathrm{B}$ and homopolymers $\mathrm{A}$, are different than those in binary homopolymer blends and copolymer blends. In the case of binary homopolymer blends one observes macrophase separation at $q=0$, whereas one observes microphase separation at finite $q=q_{\mathrm{c}}$, due to chemical connectivity in the case of copolymer blends. In ternary copolymerhomopolymer mixtures, one can have both macro and microphase separation depending on the volume fraction $\phi$ of the copolymers, and the molecular weights of the species. From equation (17), one obtains the critical

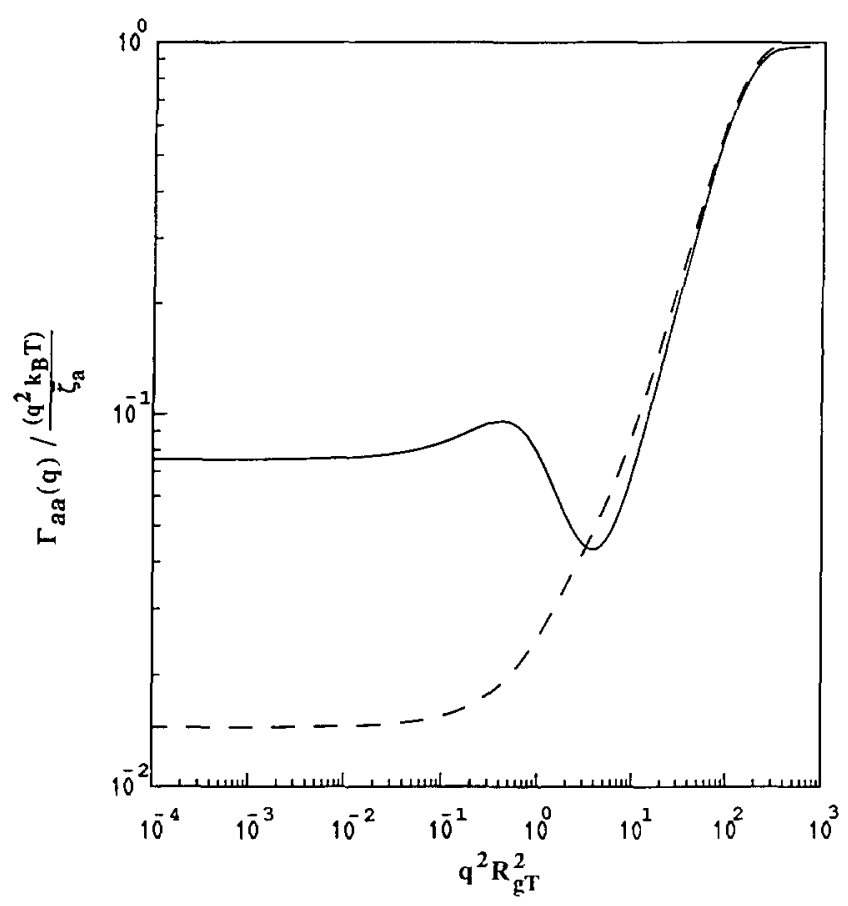

Figure 2 Theoretical variation of the normalized first cumulan $\Gamma_{\mathrm{aa}}(q) /\left(q^{2} k_{\mathrm{B}} T / \bar{\zeta}_{\mathrm{a}}\right)$, [equation (21a)], as a function of the wave number $q$ at two different volume fractions of copolymers, for the ternary mixture of copolymers $\mathrm{A}-\mathrm{B}$ in the matrix of homopolymers $\mathrm{C}=\mathrm{A}$ with number of segments $N_{\mathrm{a}}=34, N_{\mathrm{b}}=38, N_{\mathrm{c}}=221$ and segment length $a=1.6 \mathrm{~nm}:(-) \phi=0.95, \kappa=0.5 \kappa_{\mathrm{c}}^{\text {micro }} ;(-\longrightarrow) \phi=0.1$, $\kappa=0.5 \kappa_{\mathrm{c}}^{\text {macro }}$ 


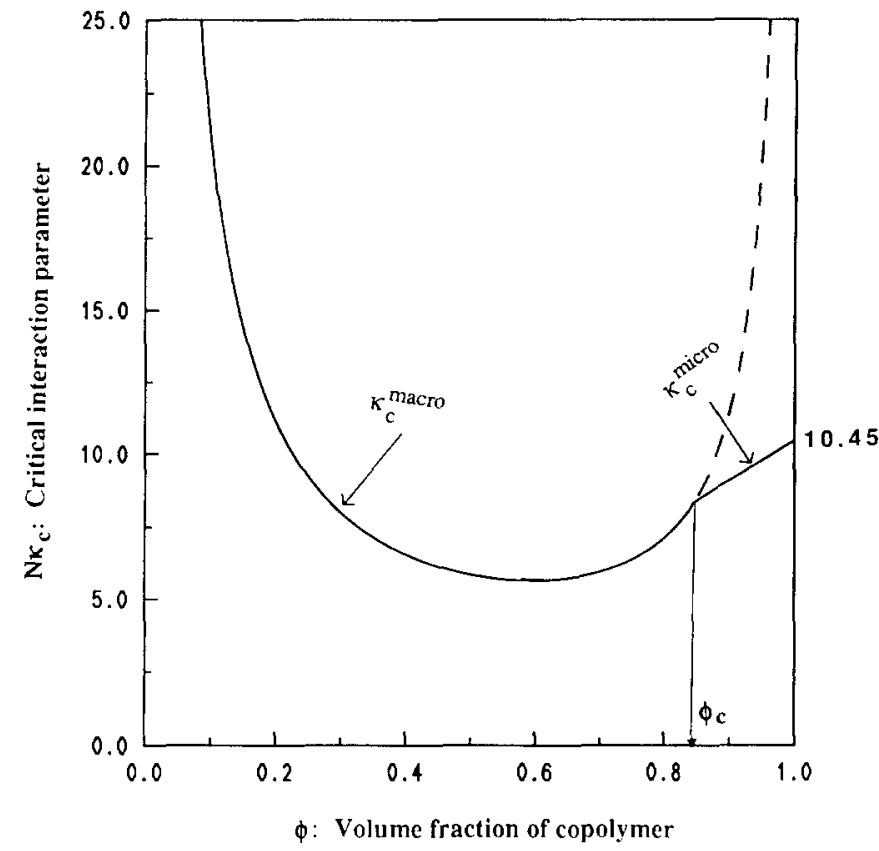

Figure 3 Phase diagram showing the stability limits for macro- and microphase separation as a function of the volume fraction of copolymer with $N_{\mathrm{a}}=N_{\mathrm{b}}=24, N_{\mathrm{c}}=104$, and $a=2.0 \mathrm{~nm}$

value $\kappa_{\mathrm{c}}$ of the interaction parameter for macro and microphase transition, by setting the denominators equal to zero, as follows:

$$
\begin{aligned}
2 \kappa_{\mathrm{c}}^{\text {macro }} & =\left.\frac{S_{\mathrm{aa}}^{\mathrm{o}}+S_{\mathrm{bb}}^{\mathrm{o}}+2 S_{\mathrm{ab}}^{\mathrm{o}}+S_{\mathrm{cc}}^{\mathrm{o}}}{S_{\mathrm{bb}}^{\mathrm{o}} S_{\mathrm{cc}}^{\mathrm{o}}+\Delta^{o}}\right|_{q=0} \\
2 \kappa_{\mathrm{c}}^{\text {micro }} & =\left.\frac{S_{\mathrm{aa}}^{\mathrm{o}}+S_{\mathrm{bb}}^{\mathrm{o}}+2 S_{\mathrm{ab}}^{\mathrm{o}}+S_{\mathrm{cc}}^{\mathrm{o}}}{S_{\mathrm{bb}}^{\mathrm{o}} S_{\mathrm{cc}}^{\mathrm{o}}+\Delta^{o}}\right|_{q=q_{\mathrm{c}}}
\end{aligned}
$$

In the case of macrophase separation, the minimum value of the interaction parameter, at which the denominator of $S_{i j}(q)$ in equation (17) vanishes, occurs at $q=0$, whereas it occurs at a finite $q=q_{\mathrm{c}}$ in the case of microphase separation. The value of $q_{\mathrm{c}}$ is, therefore, obtained by finding the minimum of the right-hand side of equation (44b) as a function of $q$. This procedure also yields the critical value, $\phi_{\mathrm{c}}$, of the volume fraction by evaluating the derivative of the right-hand side of equation (44a) or (44b) at $q=0$ because at $\phi_{c}, q_{c}=0$. Substituting the small $q$ limits of the bare system static structure factors, given in equation (22), into equation (44), we calculate the critical volume fraction $\phi_{\mathrm{c}}$ as

$$
\begin{aligned}
& \frac{f_{\mathrm{c}}^{2}}{4 f_{\mathrm{a}}^{2}}-\frac{\left(1+2 f_{\mathrm{a}}\right) f_{\mathrm{c}}}{4 f_{\mathrm{a}}} \\
& +\sqrt{\left[\frac{f_{\mathrm{c}}^{2}}{4 f_{\mathrm{a}}^{2}}-\frac{\left(1+2 f_{\mathrm{a}}\right) f_{\mathrm{c}}}{4 f_{\mathrm{a}}}\right]^{2}+\frac{f_{\mathrm{c}}^{2}\left(1-f_{\mathrm{a}}\right)}{2 f_{\mathrm{a}}^{2}}} \\
& \phi_{\mathrm{c}}=\frac{f_{\mathrm{c}}^{2}}{4 f_{\mathrm{a}}^{2}}-\frac{\left(1+2 f_{\mathrm{a}}\right) f_{\mathrm{c}}}{4 f_{\mathrm{a}}} \\
& +\sqrt{\left[\frac{f_{\mathrm{c}}^{2}}{4 f_{\mathrm{a}}^{2}}-\frac{\left(1+2 f_{\mathrm{a}}\right) f_{\mathrm{c}}}{4 f_{\mathrm{a}}}\right]^{2}+\frac{f_{\mathrm{c}}^{2}\left(1-f_{\mathrm{a}}\right)}{2 f_{\mathrm{a}}^{2}}}
\end{aligned}
$$

At $\phi_{c}$, the $\kappa_{\mathrm{c}}$ values calculated from equation (44) for macro and microphase separation become identical.

The variation of $\kappa_{\mathrm{c}}$ with the volume fraction of copolymers is presented in Figure 3. One observes that macrophase separation occurs when $\phi<\phi_{c}$, and microphase separation occurs when $\phi>\phi_{\mathrm{c}}$. In the latter case, the interaction between the two arms of diblock copolymers is dominant, and hence the mixture behaves as a copolymer melt. In Figure 4, we plotted $\phi_{c}$ as a function of $f_{\mathrm{a}}=N_{\mathrm{a}} /\left(N_{\mathrm{a}}+N_{\mathrm{b}}\right)$ for three values of $f_{\mathrm{c}}=N_{\mathrm{c}} /\left(N_{\mathrm{a}}+N_{\mathrm{b}}\right)$. One observes that $\phi_{\mathrm{c}}$ decreases at a fixed $f_{\mathrm{a}}$, when the degree of polymerization $N_{\mathrm{c}}$ of homopolymers $\mathrm{A}$ is decreased. In other words, the microphase separation takes place first at a fixed $\phi$, when the relative size $f_{\mathrm{c}}$ of the matrix homopolymers relative to the size of copolymers is reduced sufficiently.

The variation of $q_{c}$ with $\phi$ of the copolymer for three different sizes of the matrix homopolymers is presented in Figure 5. When $\phi$ approaches unity we recover earlier copolymer melt result ${ }^{7,25}$. The value of $q_{\mathrm{c}}$ is identically zero for all $\phi<\phi_{c}$, because at these volume fractions only macrophase separation is possible. It is also interesting to observe that $q_{\mathrm{c}}$ decreases from its value $q_{\mathrm{c}} R_{\mathrm{gT}} \sim 1.947$ to zero, implying an increase in the effective size of the $\mathrm{A}$ arm of the diblock copolymers, when the size of the matrix $\mathrm{C}=\mathrm{A}$ homopolymers is increased.

\section{VARIATION OF THE DECAY RATES OF THE MODES WITH $q$}

It is also interesting to investigate the variation of the relaxation frequencies $\lambda_{1}(q)$ and $\lambda_{2}(q)$, and the amplitudes $a_{1}(q)$ and $a_{2}(q)$ of the modes as a function of $q$, because they are experimentally measurable quantities. The decay rates characterize the time evolution of the dynamic scattering function in a ternary polymer blend in the one phase region. In this section, we explicitly calculate the short time decay rates by adopting Rouse dynamics to describe the bare system in the ternary polymer mixture studied by Stühn and Rennie. From

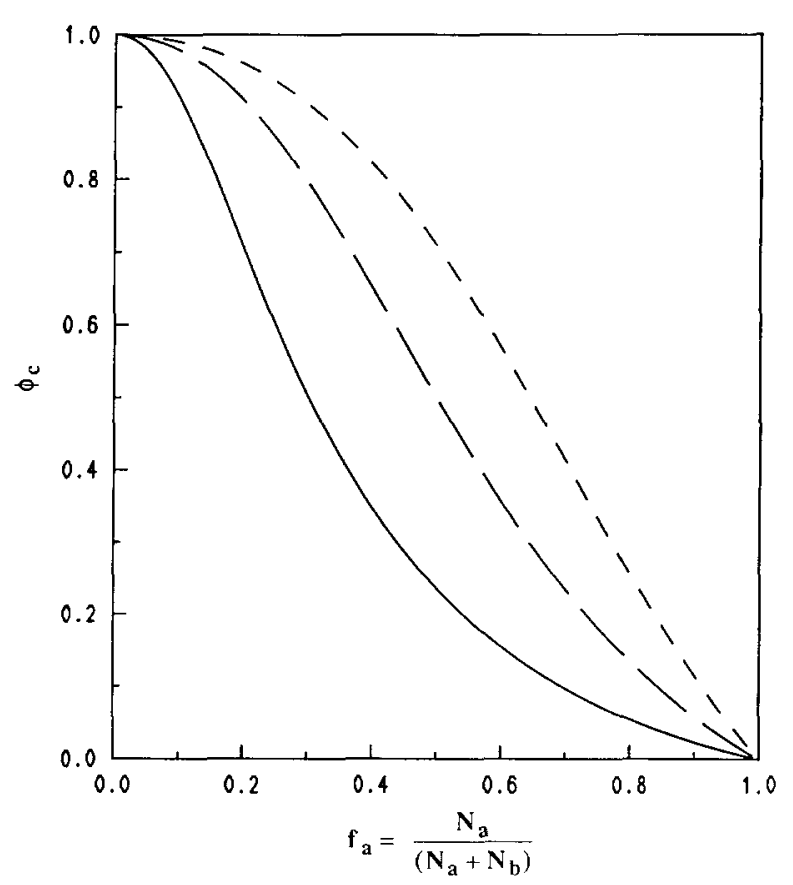

Figure 4 Theoretical variation of the critical volume fraction $\phi_{\mathrm{c}}$ of copolymers as a function of $f_{\mathrm{a}}=N_{\mathrm{a}} /\left(N_{\mathrm{a}}+N_{\mathrm{b}}\right)$ for three different values of $f_{\mathrm{c}}\left[=\left(N_{\mathrm{c}} / N_{\mathrm{a}}+N_{\mathrm{b}}\right)\right]:(\longrightarrow) 0.5 ;(--) 1.0 ;(--) 1.5$ 


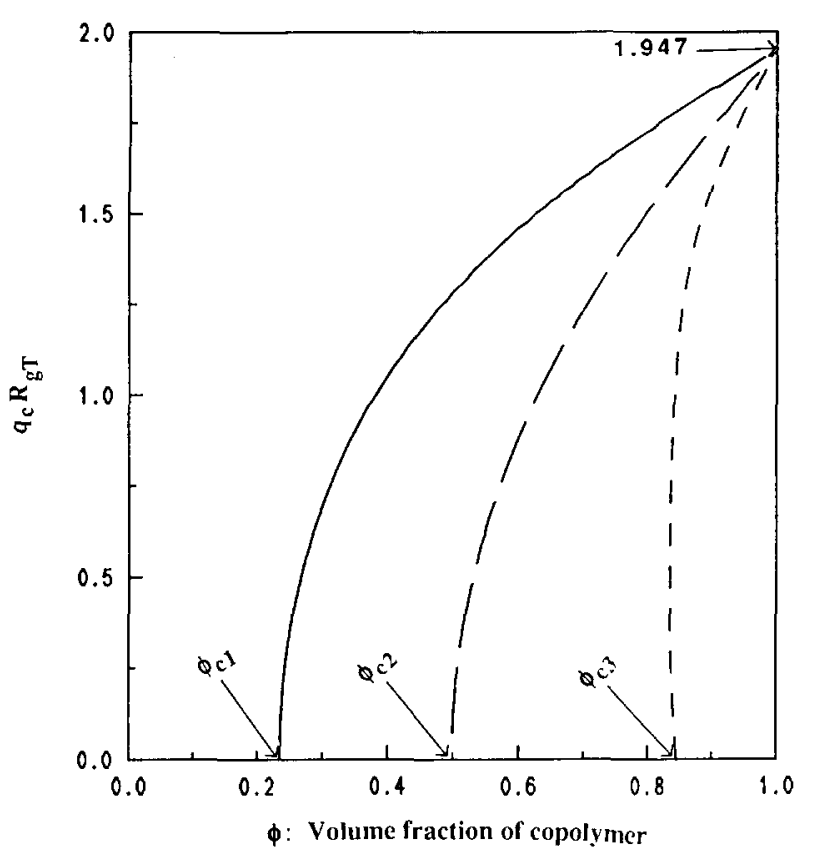

Figure 5 Theoretical variation of the critical wave number $q_{\mathrm{c}} R_{\mathrm{g}}$ as a function of the volume fraction of the copolymers for a ternary system of 50-50 diblock copolymer A-B with number of segments $N_{\mathrm{a}}+N_{\mathrm{b}}=48$ $a=2 \mathrm{~nm}$, and a matrix of homopolymers $\mathrm{C}=\mathrm{A}$ with three different molecular weights. $N_{c}:(-) 24 ;(-\longrightarrow) 48 ;(--) 104$

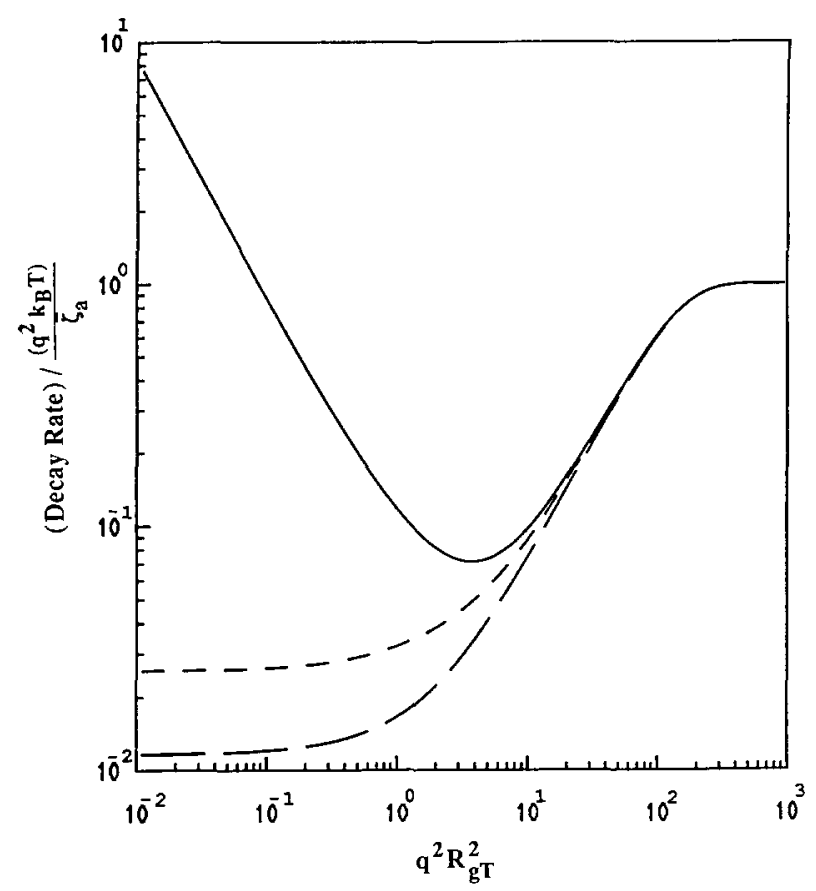

Figure 6 Theoretical variation of the normalized decay rates as a function of the wave number $q$ for the ternary system of copolymers A-B in the matrix of homopolymers $\mathrm{C}=\mathrm{A}$ characterized by $N_{\mathrm{a}}=34$, $N_{\mathrm{b}}=38, N_{\mathrm{c}}=221, \phi=0.07, a=1.6 \mathrm{~nm}$ and $\kappa=0.045$. ( - $) \lambda_{1}(q)$; $(--) \lambda_{2}(q) ;(--) \Gamma_{\mathrm{aa}}(q)$

equation (2) one obtains

$$
\begin{aligned}
& \lambda_{1}(q)=\Omega_{\mathrm{av}}+\sqrt{\Omega_{\mathrm{av}}^{2}-|\boldsymbol{\Omega}|} \\
& \lambda_{2}(q)=\Omega_{\mathrm{av}}-\sqrt{\mathbf{\Omega}_{\mathrm{av}}^{2}-|\boldsymbol{\Omega}|}
\end{aligned}
$$

where $\Omega_{\mathrm{av}}=\left(\Omega_{11}+\Omega_{22}\right) / 2$ and

$$
|\boldsymbol{\Omega}|=\left(\Omega_{11} \Omega_{22}-\Omega_{12} \Omega_{21}\right)
$$

By using equation (4), we calculate

$$
\begin{aligned}
& \Omega_{\mathrm{av}}=\frac{q^{2} k_{\mathrm{B}} T}{2 \Delta^{\circ} S_{\mathrm{cc}}^{\mathrm{o}}}\left(m_{\mathrm{aa}} S_{\mathrm{bb}}^{\mathrm{o}} S_{\mathrm{cc}}^{\mathrm{o}}+m_{\mathrm{bb}} S_{\mathrm{aa}}^{\mathrm{o}} S_{\mathrm{cc}}^{\mathrm{o}}+m_{\mathrm{cc}} \Delta^{\circ}\right. \\
&\left.-2 m_{\mathrm{ab}} S_{\mathrm{cc}}^{\mathrm{o}} S_{\mathrm{ab}}^{\mathrm{o}}-2 \kappa m_{\mathrm{bb}} S_{\mathrm{cc}}^{\mathrm{o}} \Delta^{\mathrm{o}}\right) \\
&|\boldsymbol{\Omega}|=\frac{\left(q^{2} k_{\mathrm{B}} T\right)^{2}}{\Delta^{\circ} S_{\mathrm{cc}}^{\mathrm{o}}}\left(m_{\mathrm{aa}} m_{\mathrm{bb}}-m_{\mathrm{ab}}^{2}\right) \\
& \times\left[S_{\mathrm{aa}}^{\mathrm{o}}+S_{\mathrm{bb}}^{\mathrm{o}}+2 S_{\mathrm{ab}}^{\mathrm{o}}+S_{\mathrm{cc}}^{\mathrm{o}}-2 \kappa\left(S_{\mathrm{bb}}^{\mathrm{o}} S_{\mathrm{cc}}^{\mathrm{o}}+\Delta^{\circ}\right)\right]
\end{aligned}
$$

where the $m_{i j}$ values are given by equation (5) and $\Delta^{\circ}=S_{\mathrm{aa}}^{\mathrm{o}} S_{\mathrm{bb}}^{\mathrm{o}}-S_{\mathrm{ab}}^{\mathrm{o} 2}$. In Figure 6 , the normalized decay rates are plotted as a function of $q^{2}\left(R_{\mathrm{ga}}^{2}+R_{\mathrm{gb}}^{2}\right)$. In the numerical calculations using equation (44), we assumed that the friction coefficients of $A$ and $B$ parts of copolymers and homopolymers $A$ are the same. We note that we did not have to introduce this assumption in the calculation of the first cumulant from equation (21), because the friction coefficients can be lumped into a single effective friction coefficient, as indicated by equation (26). In the small $q$ region one observes very different behaviour for $\lambda_{1}(q)$ and $\lambda_{2}(q)$. The relaxation frequency of the mode associated with the relative motion of the two arms of copolymers, which we choose to be the first mode, remains finite as $q \rightarrow 0$, and hence $\lambda_{1}(q) / q^{2}$ tends to infinity in the small $q$ limit. However, the amplitude of this mode vanishes in this limit, as we discuss below. The other mode describes the interdiffusion of the homopolymers and the copolymer molecules, and hence vanishes as $q^{2}$ as $q \rightarrow 0$. In the intermediate and high $q$ regions, both decay rates have the same qualitative behaviour, which is similar to that of the first cumulant. This is expected because for such large values of $q$, only the internal motions of the chains are probed.

The amplitudes of the modes depend on the scattering

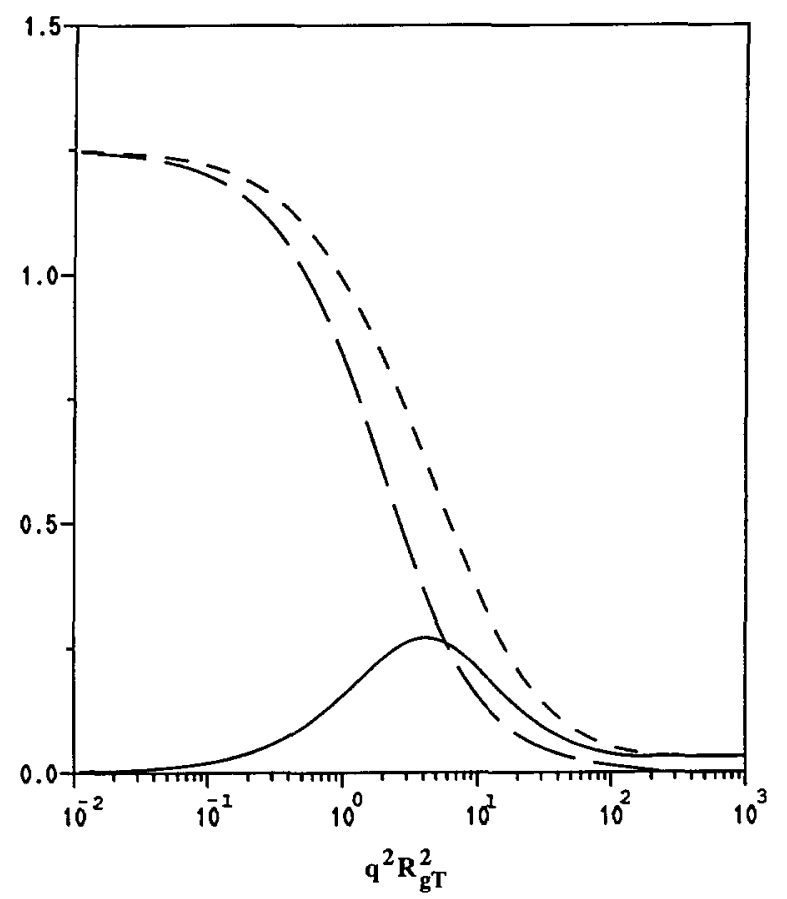

Figure 7 Variation of the amplitudes of the exponential decay modes and partial structure factor as a function of the wave number $q$ for the same ternary system described in Figure $6 .(-) a_{1}(q) ;(--) a_{2}(q)$; $(--) S_{\mathrm{aa}}(q)=a_{1}(q)+a_{2}(q)$ 
lengths of each species. In the case where the A part of the diblock copolymer is labelled, one can calculate the amplitudes by using equations ( $3 a$ ) and ( $3 b$ ). In Figure 7 , we plotted both the amplitudes and the partial structure factor $S_{\mathrm{aa}}(q)$ as a function of $q^{2}\left(R_{\mathrm{ga}}^{2}+R_{\mathrm{gb}}^{2}\right)$. One observes that $a_{1}(q)$ vanishes as $q \rightarrow 0$, and displays a maximum at a finite $q$, which depends on the size, as well as on the volume fraction, of the copolymers. On the other hand, $a_{2}(q)$ approaches $S_{\mathrm{aa}}(q)$ as $q \rightarrow 0$, which also implies that in the small $q$ limit, the decay of $S_{\mathrm{aa}}(q, t)$ is dominated by the interdiffusion process.

\section{CONCLUSIONS}

In this paper we have derived, within the framework of the RPA, analytical expressions for the relaxation frequencies and the amplitudes of the modes as a function of $q$, which are observed in dynamic light or neutron scattering experiments on a ternary polymer mixture consisting of homopolymers $\mathrm{A}$ and $\mathrm{B}$, or diblock copolymers A-B in a matrix of homopolymer C. In addition, an expression for the first cumulant of the dynamic scattering function of a labelled component is obtained for all values of $q$, including the high $q$ region where the segmental diffusion mediates the relaxation of the scattering function. With the help of the theoretical results presented, interpretation of dynamic scattering experiments on ternary mixtures have been reduced in any $q$ region to straightforward numerical calculations which can be performed perhaps more conveniently in matrix form. As an application, we have employed these results to interpret the experiment by Stühn and Rennie ${ }^{4}$, in which the first cumulant was measured with the neutron spin-echo technique in the intermediate and high $q$ regions on a mixture consisting of $\mathrm{A}-\mathrm{B}$ diblock copolymers in a matrix of A homopolymers. Using this example, we have demonstrated that one can infer the segment length and the effective friction coefficient per segment, by comparing the theoretical expression of the first cumulant obtained in this paper with the data in this $q$ region. We also studied the phase diagram for this particular mixture, which can undergo either macrophase or microphase separation depending on the volume fraction of diblock copolymers and molecular weights. We determined the spinodal line, and calculated the variation of $q_{\mathrm{c}}$ associated with microphase separation, as a function of molecular weights of the species and the volume fraction of the copolymers.

\section{ACKNOWLEDGEMENTS}

We thank Dr B. Stühn and Dr A. R. Rennie for sending us their data in Table 2 and for carefully reading the manuscript. Acknowledgement is made to the donors of the Petroleum Research Fund, administered by the American Chemical Society, for supporting this project. One of us (MT) would like to thank the Turkey Ministry of Education for financial support.

\section{REFERENCES}

1 Nemota, N., Makita, Y., Tsunashima, Y. and Kurata, M Macromolecules 1984, 17, 2629

2 Borsali, R., Duval, M., Benoit, H. and Benmouna, M Macromolecules 1987, 20, 1112; Borsali, R., Duval, M. and Benmouna, M. Macromolecules 1989, 22, 816
3 Brown, W. and Zhou, P. Macromolecules 1989, 22, 3508, 4031; Brown, W., Konack, C., Jhonsen, M. and Zhou, P. Macromolecules 1990, 23, 901

4 Stühn, B. and Rennie, A. R. Macromolecules 1989, 22, 2460

5 Akcasu, A. Z., Hammouda, B., Lodge, T. P. and Han, C. C. Macromolecules 1984, 17, 759

6 Akcasu, A. Z., Benmouna, M. and Hammouda, B. J. Chem. Phys. 1984, 80, 2762

7 Akcasu, A. Z., Benmouna, M. and Benoit, H. Polymer 1986, 27, 1935

8 Benmouna, M., Benoit, H., Duval, M. and Akcasu, A. Z. Macromolecules 1987, 20, 1107

9 Benmouna, M., Benoit, H., Borsali, R. and Duval, M. Macromolecules 1987, 20, 2620

10 Foley, G. and Cohen, C. Macromolecules 1987, 20, 1891

11 Akcasu, A. Z. and Tombakoglu, M. Macromolecules 1990, 23, 607

12 de Gennes, P. G. J. Chem. Phys. 1980, 72, 4756

13 Pincus, P. J. Chem. Phys. 1981, 75, 1996

14 Binder, K. J. Chem. Phys. 1983, 79, 6387

15 Zimm, B. H. J. Chem. Phys. 1946, 14, 164; 1948, 16, 1093

16 Benoit, H., Benmouna, M. and Wu, W. Macromolecules 1990. 23, 1511

17 Akcasu, A. Z. and Gurol, H. J. Polym. Sci., Polym. Phys. Edn 1976, 14, 1

18 Benoit, H. and Hadziioannou, G. Macromolecules 1988, 21, 1449

19 de Gennes, P.-G. 'Scaling Concepts in Polymer Physics', Cornell University Press, Ithaca, 1979, Ch. IV

20 Bates, F. S. and Hartney, M. A. Macromolecules 1985, 18, 2478

$21 \quad$ Bates, F. S. Macromolecules 1987, 20, 2221

22 Marquardt, D. W. J. Soc. Ind. Appl. Math. 1963, 11, 431

23 Bevington, P. R. 'Data Reduction and Error Analysis for the Physical Sciences', McGraw-Hill, New York, 1969

24 Hashimoto, T., Tanaka, H. and Hasegawa, H. 'Studies in Polymer Science', Vol. 2, Elsevier, Amsterdam, 1988, pp. 257 283

25 Leibler, L. Macromolecules 1980, 13, 1602

26 Golup, G. H. and Van Loan, C. F. "Matrix Computations', The Johns Hopkins University Press, London, 1989, Ch. II

\section{APPENDIX}

\section{Multicomponent mixtures consisting of two chemical species}

We consider an incompressible mixture of $N+1$ components consisting of only two kinds of chemical species A and B. One of the A homopolymers is taken to be the matrix component designated by $C$. The molecules of the $A$ and $B$ species differ from each other only in their sizes. The A and B molecules may be connected to each other to form complex molecules as copolymers, stars, even large networks, except for the component chosen as the matrix. Since the mixture consists of only A and B monomers, there is only one non-vanishing Flory interaction parameter $\kappa_{\mathrm{ab}}=\kappa$ in the system, as can be seen from equation $(8 \mathrm{c})$. The purpose of this appendix is to show that the description of such a mixture can be essentially reduced to the dynamics of a binary mixture.

The incremental number densities of the components in the Fourier space are labelled as $\rho_{j \alpha}$, where the subscript $j$ denotes a molecule belonging to species $\alpha=\mathrm{A}, \mathrm{B}$. Hence, the density vector can be written as an $N$ vector $\boldsymbol{\rho}(q)=\operatorname{Col}\left[\rho_{1 \mathrm{a}}, \rho_{2 \mathrm{a}}, \rho_{3 \mathrm{a}}, \ldots, \rho_{\mathbf{M}_{\mathrm{A}} \mathrm{a}}, \rho_{1 \mathrm{~b}}, \rho_{2 \mathrm{~b}}, \ldots, \rho_{M_{\mathrm{B}} \mathrm{b}}\right]$, where $M_{\alpha}$ is the number of molecules in the component $\alpha$. The density of the matrix component $\rho_{\mathrm{c}}$ is not included in $\rho(q)$, because it is eliminated using incompressibility constraint. The first task is to calculate the $N \times N$ static structure matrix $\mathbf{S}(q)=\left\langle\boldsymbol{\rho}(q) \boldsymbol{\rho}^{\dagger}(q)\right\rangle$, using the inverse Zimm formula given by equation (7). The reduction to a binary mixture becomes possible because the excluded volume matrix $\mathbf{v}(q)$ in this mixture can be factorized as 
a product of two rectangular matrices as

$$
\mathbf{v}(q)=\mathbf{U} \mathbf{V}^{\mathbf{T}}
$$

where

$$
\mathbf{U}^{\mathrm{T}}=\left[\begin{array}{llllllllll}
1 & 1 & 1 & \ldots & 1 & 1 & 1 & \ldots & 1 & 1 \\
0 & 0 & 0 & \ldots & 0 & 1 & 1 & \ldots & 1 & 1
\end{array}\right]
$$

$\mathbf{v}^{\mathrm{T}}=$

$$
\left[\begin{array}{cccccccccc}
\frac{1}{S_{\mathrm{cc}}^{\mathrm{o}}} & \frac{1}{S_{\mathrm{cc}}^{\mathrm{o}}} & \frac{1}{S_{\mathrm{cc}}^{\mathrm{o}}} & \ldots & \frac{1}{S_{\mathrm{cc}}^{\mathrm{o}}} & \frac{1}{S_{\mathrm{cc}}^{\mathrm{o}}} & \frac{1}{S_{\mathrm{cc}}^{\mathrm{o}}} & \ldots & \frac{1}{S_{\mathrm{cc}}^{\mathrm{o}}} & \frac{1}{S_{\mathrm{cc}}^{\mathrm{o}}} \\
0 & 0 & 0 & \ldots & 0 & -2 \kappa & -2 \kappa & \ldots & -2 \kappa & -2 \kappa
\end{array}\right]
$$

Because of this special form of $\mathbf{v}(q)$, equation (7) can be inverted by using the Sherman-Morrison-Woodbury ${ }^{26}$ formula as

$$
\mathbf{S}(q)=\mathbf{S}^{\mathfrak{o}}(q)-\mathbf{S}^{\mathfrak{o}}(q) \mathbf{U}\left[\mathbf{I}+\mathbf{V}^{\mathrm{T}} \mathbf{S}^{\mathfrak{o}}(q) \mathbf{U}\right]^{-1} \mathbf{V}^{\mathrm{T}} \mathbf{S}^{\mathfrak{o}}(q)
$$

The intensity of the scattered beam is in general given by $I(q)=\mathbf{a}^{\mathrm{T}} \mathbf{S}(q) \mathbf{a}$, where the components of a denote the contrast factors relative to the matrix. Here, we allow the monomers in different components to have different scattering lengths. If the scattering lengths of all the monomers of species A and B are the same, and denoted by ' $a$ ' and ' $b$ ', but still allowing a different scattering length ' $c$ ' for the monomers in the matrix, the intensity can be simplified as

$$
\begin{aligned}
I(q)= & (a-c)^{2} S_{\mathrm{aa}}(q)+(b-c)^{2} S_{\mathrm{bb}}(q) \\
& +2(a-c)(b-c) S_{\mathrm{ab}}(q)
\end{aligned}
$$

where $S_{\alpha \beta}(q)=\left\langle\rho_{\alpha}(q) \rho_{\beta}(-q)\right\rangle$ with $\alpha, \beta=a, b$, and denotes the structure factor in the interacting system for the total densities of the components, i.e.

$$
\rho_{\alpha}(q)=\sum_{j=1}^{M_{\alpha}} \rho_{j \alpha}(q) ; \quad \alpha=a \text { or } b
$$

and

$$
S_{\alpha \beta}(q)=\sum_{j=1}^{M_{\alpha}} \sum_{k=1}^{M_{\beta}} S_{\alpha_{j} \beta_{k}}(q)
$$

The expressions of $S_{\alpha_{j} \beta_{k}}(q)=\left\langle\rho_{\alpha_{j}}(q) \rho_{\beta_{k}}^{*}(q)\right\rangle$ in terms of the bare system static structure factors are obtained from equation (A4). The results can be written after some lengthy calculations as

$$
S_{\mathrm{aa}}(q)=\frac{S_{\mathrm{aa}}^{\mathrm{o}} S_{\mathrm{cc}}^{\mathrm{o}}+\Delta^{\mathrm{o}}-2 \kappa S_{\mathrm{cc}}^{\mathrm{o}} \Delta^{\mathrm{o}}}{S_{\mathrm{aa}}^{\mathrm{o}}+S_{\mathrm{bb}}^{\mathrm{o}}+2 S_{\mathrm{ab}}^{\mathrm{o}}+S_{\mathrm{cc}}^{\mathrm{o}}-2 \kappa\left(S_{\mathrm{bb}}^{\mathrm{o}} S_{\mathrm{cc}}^{\mathrm{o}}+\Delta^{\mathrm{o}}\right)}
$$

$$
S_{\mathrm{bb}}(q)=\frac{S_{\mathrm{bb}}^{o} S_{\mathrm{cc}}^{\mathrm{o}}+\Delta^{\mathrm{o}}}{S_{\mathrm{aa}}^{\mathrm{o}}+S_{\mathrm{bb}}^{\mathrm{o}}+2 S_{\mathrm{ab}}^{\mathrm{o}}+S_{\mathrm{cc}}^{\mathrm{o}}-2 \kappa\left(S_{\mathrm{bb}}^{\mathrm{o}} S_{\mathrm{cc}}^{\mathrm{o}}+\Delta^{\mathrm{o}}\right)}
$$

$$
S_{\mathrm{ab}}(q)=\frac{S_{\mathrm{ab}}^{\mathrm{o}} S_{\mathrm{cc}}^{\mathrm{o}}-\Delta^{\mathrm{o}}}{S_{\mathrm{aa}}^{\mathrm{o}}+S_{\mathrm{bb}}^{\mathrm{o}}+2 S_{\mathrm{ab}}^{\mathrm{o}}+S_{\mathrm{cc}}^{\mathrm{o}}-2 \kappa\left(S_{\mathrm{bb}}^{\mathrm{o}} S_{\mathrm{cc}}^{o}+\Delta^{\circ}\right)}
$$

where

$$
\begin{gathered}
S_{\alpha \beta}^{\mathrm{o}}(q)=\sum_{j=1}^{M_{\alpha}} \sum_{k=1}^{M_{\beta}} S_{\alpha_{j} \beta_{k}}^{\mathrm{o}}(q) \\
\Delta^{\mathrm{o}}=S_{\mathrm{aa}}^{\mathrm{o}} S_{\mathrm{bb}}^{\mathrm{o}}-S_{\mathrm{ab}}^{\mathrm{o}} S_{\mathrm{ba}}^{\mathrm{o}}
\end{gathered}
$$

The form of equation (A7) is the same as that of equation (17), except for the definitions of $S_{\alpha \beta}^{o}(q)$. When $M_{\mathrm{a}}=$ $M_{\mathrm{b}}=1$, they become identical. In equations (A7) and (A8), we have not specified the bare system static structure factors $S_{\alpha \beta}^{\circ}(q)$ because their form depends on the model one adapts for the chain statistics in the bare system, as well as on the connectivities among the molecules of the components. When the scattering length of the matrix is the same as the scattering length of species A, i.e. $a=c$, then the scattering intensity in equation (A5) is proportional to $S_{\mathrm{bb}}(q)$. The generalized form of $S_{\mathrm{bb}}(q)$ in equation (A7b) can also be obtained directly from equation (18), which is written for a binary incompressible mixture by defining the density of one component as $\rho_{\mathrm{a}}+\rho_{\mathrm{c}}$, and the other as $\rho_{\mathrm{b}}$.

The first cumulant of the dynamic scattering intensity is defined as

$$
\Gamma(q)=-\lim _{t \rightarrow 0} \frac{\mathrm{d} I(q, t)}{\mathrm{d} t} \frac{1}{I(q)}
$$

where the dynamic scattering intensity $I(q, t)$ is defined by

$$
I(q, t)=\mathbf{a}^{\mathrm{T}} \mathbf{S}(q, t) \mathbf{a}
$$

The short time behaviour of the dynamic scattering matrix $\mathbf{S}(q, t)$ can be expressed in terms of the mobility matrix $\mathbf{m}(q)$ defined as

$$
\mathbf{S}(q, t)=\exp \left[-q^{2} k_{\mathrm{B}} T \mathbf{m}(q) \mathbf{S}(q)^{-1} t\right] \mathbf{S}(q)
$$

Hence, using equations (A10) and (A11) in equation (A9), one obtains

$$
\Gamma(q)=q^{2} k_{\mathrm{B}} T \frac{\mathbf{a}^{\mathrm{T}} \mathbf{m}(q) \mathbf{a}}{\mathbf{a}^{\mathrm{T}} \mathbf{S}(q) \mathbf{a}}
$$

The components of $\mathbf{m}(q)$ are expressed in terms of the mobilities in the bare system by equation (5). Since the particular mixture considered in this appendix consists of only two species A and B, the bare mobilities can be expressed in terms of the segmental friction coefficients $\zeta_{\mathrm{a}}$ and $\zeta_{\mathrm{b}}$ of these species, with considerable simplification. The diagonal and off-diagonal elements follow from equations (5a) and (5c) as

$$
\begin{array}{r}
m_{\alpha_{i} \alpha_{i}}=\frac{\phi_{\alpha_{i}}}{\zeta_{\alpha}}\left(1-\frac{\phi_{\alpha_{i}} \zeta_{\mathrm{a}} \zeta_{\mathrm{b}}}{\zeta_{\alpha} \zeta}\right) ; \quad \alpha=a \text { or } b \quad \text { (A13a) } \\
m_{\alpha_{\mathrm{i}} \beta_{j}}=-\frac{\phi_{\alpha_{i}} \phi_{\beta_{j}}}{\bar{\zeta}} \frac{\zeta_{a} \zeta_{\mathrm{b}}}{\zeta_{\alpha} \zeta_{\beta}} ; \quad i \neq j, \alpha=a \text { or } b \text { and } \beta=a \text { or } b
\end{array}
$$

where $\phi_{\alpha_{i}}$ is the volume fraction of the $i$ component belonging to species ' $\alpha$ ', $a$ or $b$, and $\bar{\zeta}$ is the weighted average of the friction coefficients, and defined by

$$
\bar{\zeta}=\left(\phi_{\mathrm{a}}+\phi_{\mathrm{c}}\right) \zeta_{\mathrm{b}}+\phi_{\mathrm{b}} \zeta_{\mathrm{a}}
$$

where $\phi_{\alpha}$ is the volume fraction of the monomers belonging to ' $\alpha$ ', namely

$$
\phi_{\alpha}=\sum_{j=1}^{M_{\alpha}} \phi_{\alpha_{j}}
$$

we note that $\phi_{\mathrm{a}}$ does not include the volume fraction $\phi_{\mathrm{c}}$ of the matrix component. Hence, $\phi_{\mathrm{a}}+\phi_{\mathrm{b}} \neq 1$, whereas $\phi_{\mathrm{a}}+\phi_{\mathrm{b}}+\phi_{\mathrm{c}}=1$.

The expression $\Gamma(q)$ simplifies when the scattering lengths of all the monomers of species A and B are the 
same, and denoted by ' $a$ ' and ' $b$ ', but still allowing a different scattering length ' $c$ ' for the monomers in the matrix : the first cumulant simplified to

$\Gamma(q)=q^{2} k_{\mathrm{B}} T$

$\times \frac{(a-c)^{2} m_{\mathrm{aa}}+(b-c)^{2} m_{\mathrm{bb}}+2(a-c)(b-c) m_{\mathrm{ab}}}{(a-c)^{2} S_{\mathrm{aa}}(q)+(b-c)^{2} S_{\mathrm{bb}}(q)+2(a-c)(b-c) S_{\mathrm{ab}}(q)}$

where $S_{\alpha \beta}(q)$ with $\alpha, \beta=a, b$ is given in equation (A7), and $m_{\alpha \beta}$ is defined as:

$$
m_{\alpha \beta}=\sum_{j=1}^{M_{z}} \sum_{k=1}^{M_{\beta}} m_{\alpha_{j} \beta_{k}}
$$

which can be calculated by substituting equation (A13) into equation (A16) as :

$$
\begin{aligned}
& m_{\mathrm{aa}}=\frac{\phi_{\mathrm{a}}\left(1-\phi_{\mathrm{a}}\right)}{\bar{\zeta}_{\mathrm{a}}} \\
& m_{\mathrm{bb}}=\frac{\phi_{\mathrm{b}}\left(1-\phi_{\mathrm{b}}\right)}{\bar{\zeta}_{\mathrm{b}}}
\end{aligned}
$$

and

$$
m_{\mathrm{ab}}=-\frac{\phi_{\mathrm{a}} \phi_{\mathrm{b}}}{\bar{\zeta}}
$$

where $\bar{\zeta}_{\mathrm{a}}$ and $\bar{\zeta}_{\mathrm{b}}$ are given by equations (26) and (36). The expression of $\Gamma(q)$ in equation (A15) constitutes a generalization of equations (21a) and (21b). Indeed, when the scattering lengths of the monomers in species $\mathrm{B}$ and the matrix are the same, i.e. $b=c$, equation (A15) reduces to

$$
\Gamma(q)=q^{2} k_{\mathrm{B}} T \frac{m_{\mathrm{aa}}}{S_{\mathrm{aa}}(q)}
$$

and when $a=c$, to

$$
\Gamma(q)=q^{2} k_{\mathrm{B}} T \frac{m_{\mathrm{bb}}}{S_{\mathrm{bb}}(q)}
$$

In the special case $M_{\mathrm{a}}=M_{\mathrm{b}}=1$, i.e. for a threecomponent system, equations (A18a) and (A18b) become identical with equations (21a) and (21b). 